\title{
15th Italian Congress of Neuroepidemiology
}

Tricase, Italy, February 23-24, 2018

\section{Abstracts}

Editors

E. Beghi, Milan, Italy

M. Leone, San Giovanni Rotondo, Italy

G. Logroscino, Tricase, Italy 
1

\section{Patient Values and Preferences: Findings from the EAN Guideline on Palliative Care of People with Severe Multiple Sclerosis}

Andrea Giordano', Sascha Köpke², Simone Veronese ${ }^{3}$, Anne C. Rahn ${ }^{4}$, Ingo Kleiter ${ }^{5}$, Birgit Basedow-Rajwich ${ }^{5}$, Arianna Fornari ${ }^{1}$, Mario A. Battaglia ${ }^{6}$, Jelena Drulovic ${ }^{7}$, Liesbeth Kooij ${ }^{8}$, Johan Koops ${ }^{8}$, John Mens', Edwin R. Meza Murillo ${ }^{9}$, Ivan Milanov ${ }^{10}$, Ron Milo ${ }^{11}$, Francesco Patti ${ }^{12}$, Tatjana Pekmezovic ${ }^{7}$, Jaume Sastre-Garriga ${ }^{9}$, Eli Silber ${ }^{13}$, Janine Vosburgh ${ }^{14}$, Raymond Voltz ${ }^{15}$, Jette Bay ${ }^{16}$, David J Oliver ${ }^{17}$, Alessandra Solari ${ }^{1}$

${ }^{1}$ Foundation IRCCS Neurological Institute C. Besta, Milan, Italy; ${ }^{2}$ University of Lübeck, Lübeck, Germany; ${ }^{3}$ FARO Charitable Foundation, Turin, Italy; ${ }^{4}$ University of Hamburg, Hamburg, Germany; ${ }^{5}$ Marianne-Strauß-Klinik, Berg, Germany; ${ }^{6}$ University of Siena, Siena, Italy; ${ }^{7}$ University Hospital of Belgrade, Belgrade, Serbia; ${ }^{8}$ Nieuw Unicum, Zandvoort, The Netherlands; ${ }^{9}$ MS Centre of Catalonia (Cemcat), University Hospital Vall d'Hebron Barcelona, Spain; ${ }^{10}$ University of Sofia, Sofia, Bulgaria; ${ }^{11}$ Barzilai Medical Center, Ashkelon, Israel; ${ }^{12}$ University Hospital Policlinico Vittorio Emanuele, Catania, Italy; ${ }^{13}$ King's College Hospital, London, UK; ${ }^{14}$ Israel Multiple Sclerosis Society, Tel Aviv, Israel; ${ }^{15}$ University Hospital Cologne, Cologne, Germany;

${ }^{16}$ Multiple Sclerosis International Federation, People with MS

Advisory Committee, London, UK; ${ }^{17}$ University of Kent, Rochester, UK

Objective: To engage multiple sclerosis (MS) patients and caregivers in definition of the key questions to be answered in the European Academy of Neurology (EAN) Guideline on Palliative Care of People with Severe MS. Here we describe the results of this process, and the main challenges encountered.

Background: The incorporation of patient values into individual clinical decisions, a fundamental of shared decision making, also applies to clinical practice guideline development. The ultimate aim is to address the issues that are important to health consumers, and sensitive to their values and preferences. Consequently, professional societies and other guidelines producers have recognized the need to involve consumers in all the phases of guideline development. An initial and fundamental guideline phase is the formulation of the clinical questions, specifying the population, intervention, comparator, and outcomes (PICO).

Methods: A mixed methods approach was used: 1) International online survey launched by the national MS societies, after pilot testing/debriefing on $20 \mathrm{MS}$ patients and 18 caregivers. 2) Focus group meetings (FGMs) of Italian and German MS patients and caregivers.
Results: 1) Of 1199 participants, 951 (79\%) completed the whole survey, and 934 from seven countries were analyzed: 751 (80\%) were MS patients (74\% women, mean age 46.1 ) and 183 (20\%) caregivers $(36 \%$ spouses/partners, $72 \%$ women, mean age 47.4). Participants agreed/strongly agreed on inclusion of the nine pre-specified topics (from $89 \%$ for 'advance care planning' to $98 \%$ or 'multidisciplinary rehabilitation'), and fewer than 5\% answered 'I prefer not to answer' to any topic. Free comments were 569: 182 (32\%) on pre-specified topics, $227(40 \%)$ on additional topics (16 guideline-pertinent), and 160 (28\%) on outcomes. 2) Five FGMs (three of MS patients, two of caregivers, overall 35 participants) corroborated the survey findings, and helped to identify patientimportant outcomes.

Conclusion: Consumer involvement was resource and time intensive, but rewarding. It was key for the formulation of the guideline questions, and for the identification of patient-important outcomes. In particular free comments from several participants concerned sensitive issues which were purposely excluded from the pre-specified topics.

Acknowledgments: This is a joint initiative of the EAN, the European Association for Palliative Care (EAPC), the European Network for Best Practice and Research in MS Rehabilitation (RIMS), and has been endorsed by the European Committee for Treatment and Research in MS (ECTRIMS) by the MS International Federation (MSIF).

\section{2}

Sex-Related Differences of Fetal-Maternal Cross-Talk Modify Phenotypic Characteristics in Women with Multiple Sclerosis

\section{Alessia Bianchi, Paolo Ragonese, Sabrina Realmuto, Giulia Vazzoler, Giovanni Savettieri, Giuseppe Salemi}

Department of Experimental Biomedicine and Clinical Neurosciences, University of Palermo, Palermo, Italy

Objective: To investigate the possible role of the sex of the fetus in determining different disease characteristics among women affected by multiple sclerosis (MS).

Background: Gender-related differences in MS incidence and disease characteristics are usually attributed to hormone-dependent factors. However, several studies recently proposed the disease characteristics could be influence by a complex fetal-maternal interaction, which seems to be also related to the sex of the fetus.

Methods: We included 322 women affected by MS according to $\mathrm{McD}$ onald revised criteria, who referred to the outpatient service for MS and related diseases of the Neurological Department of the University of Palermo. By means of a structured question-

\section{KARGER}

(c) 2018 S. Karger AG, Basel 
naire, we collected information about disease course and fertile life characteristics, including the sex of the fetuses in pregnancies occurred before or after the diagnosis of MS. We calculated differences between subgroups of the cohort according to the following variables: age at onset, number of relapses within the first 3 years of disease, complete recovery after the relapses, time from pregnancy to the first relapse, rate of relapses during the postpartum period, frequency of oligoclonal bands (OCBs) in the cerebrospinal fluid (CSF) at diagnosis. Calculations were made comparing 3 subgroups of patients: MS women who did not have pregnancies before the onset of the disease, MS women who had a male child before onset and MS women who had a female child before onset.

Results: We included 322 women affected by MS, 136 of whom had a pregnancy before disease onset. Mean age at disease onset was 29.5 years (26.5 in women with no previous pregnancy, 36.4 in those with a female child, 36.2 in those with a male child). Relative risk of symptoms onset within the first year after delivery was 2.5 times higher in women who had a pregnancy with a male child compared to women who had a female child (95\% CI 1.00$6.24 ; \mathrm{p}=0.04)$. Time from pregnancy to the first symptom was longer in women who had a female child (96 months) compared to those who had a male child $(92.5$ months; $\mathrm{p}<0.05)$. There was a higher frequency of OCBs in CSF of women who had a female child compared to those with a male child $(96 \%$ vs. $81.7 \%$; $\mathrm{p}=$ 0.016).

Conclusion: This study supported the hypothesis that the immune cross-talk between the mother and fetus is modified by the sex of the fetus, and may consequently contributes to determine different clinical features in women affected by MS.

\section{3}

\section{Flynn Effect and Intellectual Disability: Clinical and Forensic Observations from a Clinical Case}

\section{A. De Giorgi, S. Lancia, A. Leo \\ Presidio di Riabilitazione Funzionale "Istituto Santa Chiara" Lecce e Scuola di Specializzazione in Psicoterapia cognitivo- Comportamentale a Indirizzo Neuropsicologico, Lecce, Italy}

Objective: To point out the impact of the Flynn effect on neuropsychological examination and the effect it may have in diagnosis (e.g. intellectual disability).

- To demonstrate the impact it can have in the medical-legal assessment.

Background: The term "intellectual disability" is now the equivalent of "intellectual development disorders" (adopted both in the DSM-5 and ICD-11 draft).

In accordance with the actual psychiatric orientation, the overall diagnostic judgement is formulated considering the clinical history, clinical objectivity, and the functional impact, rather than strict operational diagnostic criteria.

J.R. Flynn (1984), reported the results of studies conducted in the USA in which, the same individuals, have been sequentially assessed, with different versions of the same intelligence test, noting that the results obtained from the most recent version were systematically lower than those obtained in the earlier one.
Flynn's studies underlined that over the course of 46 years, the average IQ level of the USA population increased to the extent of 13.8 IQ points (33 points per year).

Other studies confirmed Flynn's observations and lead to the conclusion that the average IQ, has been increasing in a regular way for about 50 years. This phenomenon is now known as the Flynn effect.

The development of new regulatory groups occurs approximately every $10-15$ years. During that time, the population's characteristics change, while the normative references remain the same.

There is no single factor responsible of the Flynn effect: the decreased household members; the increased use of the technology; the increase in means to deal with education; the bio-environmental conditions; and the fact that the population is subjected to intelligence tests (creating a form of training). All these factors, are likely to be the basis of the Flynn effect.

New normative reference should be developed at regular intervals to correctly interpret the results.

Method: A clinical case has been studied to show possible implications of the Flynn effect.

Results: A. (62 years) arrived in our institute with a diagnosis of mild mental retardation. The test we administered (WAIS IV), gets an IQ score of 71 .

After the evaluation, he was deposed for a compromise of the ability to adequately address his own interests and needs as there is a significant impairment in the ability to carry out daily life activities autonomously. The diagnostic conclusion was: "Intellectual disability of moderate degree" with the inability to carry out activities of daily life and low participation in social life.

Conclusions: This study makes us reflect on how using the diagnoses properly. A subject, who in the past received a diagnosis of mental retardation, can now benefit from a non-real improvement. It can be inferred the impact that the Flynn effect can have on the medical-legal aspects, and all the repercussions in the forensic field.

\section{4}

\section{Epidemiological Features and Persistence to Disease Modifying Therapies in a Population of Multiple Sclerosis Patients from Three Provinces of Veneto Region}

\author{
A. Marangi ${ }^{1}$, G. Farina ${ }^{1}$, S. Forlivesi ${ }^{2}$, A. Gajofatto ${ }^{1}$, V. Vicenzi ${ }^{1}$ \\ M. Calabrese ${ }^{1}$, L. Deotto ${ }^{2}$, F. Marchioretto ${ }^{3}$, A. Forgione ${ }^{4}$, \\ G. Stenta ${ }^{5}$, M. Vianello ${ }^{6}$, M.D. Benedetti
}

${ }^{1}$ UOC Neurologia B, AOUI Verona, ${ }^{2}$ UOC Neurologia A, AOUI Verona, ${ }^{3} \mathrm{UOC}$ Neurologia, Ospedale Sacro Cuore Don Calabria di Negrar (VR), ${ }^{4}$ UOC Neurologia, Ospedale Pederzoli, Peschiera del Garda (VR), ${ }^{5}$ UOC Neurologia, Ospedale San Bortolo, Vicenza, ${ }^{6}$ UOC Neurologia, Ospedale Santa Maria di Ca' Foncello, Treviso, Italy

Objective: To describe the epidemiological characteristics and drug strategies in MS in an observational study of three provinces of Veneto Region covering a population of 2,675,144 inhabitants.
Neuroepidemiology 2018;50:74-103

DOI: $10.1159 / 000487692$ 15th Italian Congress of Neuroepidemiology, Tricase 2018 
Background: The therapeutic armamentarium in Multiple Sclerosis (MS) has grown considerably over the last 15 years, with more than 10 drugs available among first and second line therapies. Persistence to drug therapies in MS represents a critical point, since low persistence carries a risk for negative clinical outcomes.

Methods: We collected demographic and clinical data, DMTs history, and MRI features of patients regularly followed in the period January 2013 to December 2017 in the MS Centers of Verona, Treviso and Vicenza areas. McDonald diagnostic criteria and Lublin disease course classification were used to define clinical aspects, and Expanded Disability Status Scale (EDSS) to score disability. The outcome was persistence in treatment at 24 months, analysed by drug and time of therapy onset. Statistical analyses were performed by using IBM SPSS v. 21 .

Results: Data were available for 3018 patients, $66.1 \%$ of which were females. Mean age of patients was 47.9 years \pm 12.33 (range 15-87 years), with a median disease duration of 14 years. $73.8 \%$ of patients were diagnosed with the relapsing remitting variant of MS, while $20 \%$ were classified as progressive ( $13.7 \%$ secondary progressive, $6.4 \%$ primary progressive). $47.5 \%$ of patients had EDSS score $\leq 2$, while $15.6 \%$ had an EDSS $\geq 6$. Benign variants, defined by an EDSS score $\leq 2$ after $\geq 10$ years of disease course, were detected in the $19 \%$ of patients. At the last follow up, $64.8 \%$ of patients were on treatment (data available for 2661 patients), $73 \%$ with a first line and $27 \%$ with a second line drug. Among first line therapies, the most prescribed drug was dimethylfumarate (25\%). Out of 1034 cases who started DMDs since $2011,57 \%$ discontinued the therapy within 24 months due to side effects, lack of efficacy or other reasons (Log Rank $\mathrm{p}=$ 0.080 ). Prevalent reasons to quit were safety protocol for natalizumab, both side effects and inefficacy for fingolimod and azathioprine, early side effects for the new oral treatments, especially teriflunomide.

Conclusions: More than half of patients treated since 2011 with a DMT in this large observational study discontinued drug within 24 months. There is a need for a better definition of the ideal therapeutic strategy for the different groups of MS cases.

\section{5 \\ Changing Concepts of Cognitive Impairment, Decline and Dementia in Populations}

\section{Carol Brayne}

Cambridge Institute of Public Health, University of Cambridge, London, UK

In the mid to later decades of the last century interest in cognition and dementia over the lifecourse has received intense interest. Earlier studies of cognition across the lifecourse had a strong focus on volunteer or college student samples. Analysis of those who persisted in such studies suggested that ageing was not associated with substantial decline. However in parallel clinical studies and those based in populations provided clear evidence of increasing proportions of the population at older ages meeting contemporary diagnostic criteria for dementia. During more recent decades the examination of cognition across time has been pur- sued in population representative studies allowing an integration of seemingly conflicting results. Amongst these studies are some from the UK. These started in the 1980s and 1990s and include follow up over many years, as well as recruitment of new generational cohorts. These have provided the insights that those who are responders and survivors do indeed have better trajectories of cognition over time and amongst these would be those similar to the volunteer cohorts. These studies retain more of the population, and also are able to correct to a large extent for the bias created by drop out. This reveals that there is substantial cognitive decline across age, and that this accelerates in the latter stages of life with the well known but relatively neglected phenomenon of terminal decline. The binary approach to cognitive decline and the definition of dementia is valuable for clinical practice but it tends to conceal the huge complexity of dementia, cognitive decline and ageing. To add to this complexity new generational studies have shown considerable reduction in age specific dementia prevalence and incidence, as well as improved life expectancies with cognitive health, even in the presence of major increases in life expectancy in the population.

\section{6}

Prevalence of Different Patterns of Amyloidosis and Neurodegeneration in a Tertiary Referral Center for Neurodegenerative Diseases

\author{
Chiara Zecca', Rosanna Tortelli', Rosa Capozzo', \\ Maria Paola Mogavero' ${ }^{1}$, Roberta Barone ${ }^{1}$, Maria Rosaria Barulli', \\ Cinzia Musarò' , Petronilla Battista ${ }^{1}$, Marco Piccininni', \\ Gaetano Scianatico ${ }^{1}$, Marco Musio ${ }^{1}$, Vincenzo Brescia ${ }^{2}$, \\ Giancarlo Logroscino ${ }^{1}$
}

'Unit of Neurodegenerative Disease, Department of Clinical Research in Neurology, University of Bari "Aldo Moro" at "Pia Fondazione Card. G. Panico", Tricase, Lecce, Italy; ${ }^{2}$ Unit of Laboratory Medicine, "Pia Fondazione Card.G. Panico", Tricase, Lecce, Italy

Objective: To evaluate the frequency of different patterns of amyloidosis and tau-related neurodegeneration in a two-year clinical based sample, applying a new classification system (A/N system) for biomarkers of amyloidosis and neurodegeneration.

Background: Biomarkers of brain amyloidosis and tau-related neurodegeneration/synaptic dysfunction are featured in the most recent diagnostic criteria for $\mathrm{AD}$ and other dementia conditions. A new classification system based on cerebrospinal fluid (CSF) or imaging measures of amyloid (A) and neurodegeneration $(\mathrm{N})$ has been proposed. Both amyloidosis $(\mathrm{A})$ and neurodegeneration $(\mathrm{N})$ can be present $(+)$ or absent $(-)$ identifying four possible patterns: $\mathrm{A}-\mathrm{N}-; \mathrm{A}+\mathrm{N}-; \mathrm{A}-\mathrm{N}+\mathrm{A}+\mathrm{N}+$.

Methods: We analysed the frequency of the four patterns of A and $\mathrm{N}$, independently from the clinical diagnosis, in 305 outpatients (154 men; mean age 66 years, range $40-84$ years) of the Unit of Neurodegenerative Diseases, Department of Clinical Research in Neurology, University of Bari "Aldo Moro" at "Card G. Panico" Hospital, over the period 2016-2017. CSF biomarkers ( $\beta$-Amyloid1-42 and Tau) were used to assess the A+/A- and the $\mathrm{N}+/ \mathrm{N}-$. Based on the results of the CSF biomarker measure- 
ments, each patient was assigned to one of the four patterns of A and $\mathrm{N}$.

Results: 133 subjects (44\%) were classified as A-N-, 55 (18\%) as $\mathrm{A}+\mathrm{N}-, 31(10 \%)$ as $\mathrm{A}-\mathrm{N}+$. and $86(28 \%)$ as $\mathrm{A}+\mathrm{N}+\mathrm{A}-\mathrm{N}-$ was the most common pattern in men $(53 \%)$, while $\mathrm{A}+\mathrm{N}+$ was the most common pattern in women $(37 \%)$. A-N+ was the less frequent pattern in both gender groups (10\%). The prevalence of the extreme patterns $(\mathrm{A}+\mathrm{N}+$ and $\mathrm{A}-\mathrm{N}-)$ was influenced by age: the highest frequency of $A+N+$ was from age 65 up to age 80 , while the prevalence of $\mathrm{A}-\mathrm{N}-$ had a peak at age 65 and then decreased in older ages. Prevalence of intermediate patterns $(\mathrm{A}+\mathrm{N}-$ and $\mathrm{A}-$ $\mathrm{N}+$ ) was not influenced by age. The most frequent clinical diagnosis in the $\mathrm{A}+\mathrm{N}+$ and $\mathrm{A}+\mathrm{N}-$ groups was Alzheimer's Disease (54\% and $34 \%$ respectively), in the $\mathrm{A}-\mathrm{N}+$ group was Frontotemporal Dementia (23\%), and in the A-N- group was Mild Cognitive Impairment (25\%).

Conclusion: This study provides estimates of the frequency of amyloidosis and tau-related neurodegeneration in a clinical-based sample of a tertiary referral Center for neurodegenerative diseases. We observed a substantial proportion of A-N- cases in our cohort. Further studies are needed to clarify the pathophysiological mechanisms underling neurodegeneration beyond the amyloid/tau hypothesis.

\section{7}

\section{Use of Antidepressants and the Risk of Parkinson's Disease in the Local Health Trust of Bologna: A Historical Cohort Study}

\section{Zenesini, E. Baldin, L. Vignatelli, C. Fonti, G.Bonavina, G.Calandra, S. Cevoli, G. Fabbri, R. Ferrara, A. Gabellini, M. Guarino, S. Nassetti, R. Pantieri, G. Samoggia, T. Sacquegna, C. Scaglione, E. Stivanello, R. Rinaldi, P. DeCarolis, P. Cortelli, R. D'Alessandro}

IRCCS - Istituto delle Scienze Neurologiche, Bologna - Servizio di Epidemiologia e Biostatistica, Bologna, Italy

Objective: To investigate the association between the use of antidepressants and risk of subsequent Parkinson's disease (PD), both diagnosed clinically and detected through administrative database in the Local Health Trust of Bologna.

Background: Depression is considered one of the prodromal symptoms of PD along with sleep disorders, hyposmia and constipation.

Methods: Historical cohort study with use of antidepressants as exposure and PD onset as outcome. The cohort considered consisted of inhabitants of Bologna aged $\geq 35$ years in 2005; those who had used antidepressants in the previous 3 years were excluded. We applied two approaches. In the first study design (PD-ParkLink), we used the ParkLink system to detect the disease onset. ParkLink is a research study including patients with a clinical diagnosis of PD residing in Bologna. In the second study design ( $P D$-Pharm) we applied an administrative data algorithm to detect the PD onset: subjects that used levodopa or dopamine agonists for at least 365 days in 1 year. In both designs those subjects that used antidepressants for at least 180 consecutive days in 1 year were considered exposed. Hazard ratios (HR) and
95\% confidence interval (CI) were estimated with Cox proportional hazards models, using exposure as time dependent variable and adjusted for age, gender, use of medical care and comorbidities.

Results: In the PD-ParkLink design we observed from 2006 to $2016,199,255$ person-years (py) in the exposed and $4,287,018$ py in the non-exposed with adjusted $\mathrm{HR}=1.6$ (CI 1.2-2.2). The association was stronger for men (HR 2.3, CI 1.5-3.3) compared to women (HR 1.2, CI 0.7-2.0), for subjects $\leq 65$ years of age (HR 2.1, CI 1.4-3.2) vs. $>65$ years (HR 1.5, CI 0.9-2.2) and for those with less comorbidities. In the PD-Pharm design, we observed 197,062 py in the exposed and 4,282,059 py in the non-exposed with an adjusted $\mathrm{HR}=4.0$ (CI 3.4-4.6). We found a stronger association for men (HR 4.3, CI 3.5-5.4) compared to women (HR 3.8, CI $3.2-4.4$ ) and in those $\leq 65$ years of age (HR 5.9, CI 3.4-10.2) than $>65$ years of age (HR 3.8, CI 3.3-4.5). We found significant associations up to 3 years with PD-ParkLink and up to 2 years with PD-Pharm.

Conclusion: The use of antidepressants is associated with the subsequent development of PD. Our findings confirm that depression precedes the onset of motor symptoms in $\mathrm{PD}$. The association is observed up to 3 years before, stronger for younger patients, who are men and have less comorbidities.

\section{8 Epidemiology of Mitochondrial Hereditary Optic
Neuropathies (LHON) Progressive External
Ophthalmoplegia from the ER-Mito Project}

C. Fonti, R. D'Alessandro, V. Di stasi, C. La Morgia, R. Liguori, M.L. Valentino, V. Carelli, on behalf of ER-Mito study group (Regione Emilia Romagna Programma di Ricerca Regione Università 2010-2012)

IRCCS - Istituto delle Scienze Neurologiche, Bologna - Servizio Statistica epidemiologia - Azienda Usl di Bologna, Italy

Objective: To estimate the prevalence of MitD in the ERR 2) to estimate the awareness on MitD among the Neurological Services of the ERR 3) to establish shared diagnostic and therapeutic pathways.

Background: In 1988, mitochondrial myopathy with CPEO and LHON were the first two disorders to be associated with pathogenic defects of mtDNA. However, the only epidemiological data on mtDNA diseases concern only LHON in the North East of England. There are no other worldwide findings on other mtDNA diseases.

Methods: An alert system among neurology centers of the ERR was set up, asking to identify all patients with clinical features suggestive of mtDNA disease. For each center a researcher was asked to review the clinical records of both out- and in patients for the preceding two years and to record the new incident cases in a dedicated database. Further, the researcher collected information about possible cases by asking to the ophthalmologists of these services. In the Bologna Local Health Unit an intensive active search was performed both by personally contacting all neurologists and ophthalmologists and with periodic meetings to discuss about CPEO and LHON.
$78 \quad$ Neuroepidemiology 2018;50:74-103

DOI: $10.1159 / 000487692$ 15th Italian Congress of Neuroepidemiology, Tricase 2018 
The prevalence (confidence interval - CI 95\%) was calculated for both CPEO and LHON cases in the ERR area included by June 2017.

Results: CPEO. 35 subjects were included. Crude prevalence (per 100,000 residents) was 2.28 (95\% CI 1.44-3.42) in Bologna, 0.97 (CI 0.30-2.19) in Reggio Emilia, 0.57 (CI 0.69-2.07) in Ferrara, 0.56 (CI 0.06-1.83) in Forlì-Cesena, 0.29 (CI 0.007-1.65) in Rimini, 0.28 (CI 0.03-1.03) in Modena. No subjects were recorded from Ravenna Parma and Piacenza. LHON. 58 subjects were included. Crude prevalence (per 100,000 residents) was 2.57 (95\% CI 1.18-4.89) in Ferrara, 2.47 (CI 1.60-3.65) in Bologna, 1.39 (CI 0.38-3.57) in Piacenza, 0.88 (CI 0.18-2.59) in Rimini, 0.76 (CI 0.15-2.22) in Forlì-Cesena, 0.76 (CI 0.15-2.23) in Ravenna, 0.75 (CI 0.20-1.92) in Reggio Emilia, 0.67 (CI 0.141.96) in Parma, 0.57 (CI 0.15-1.46) in Modena.

Conclusion: Our preliminary data show a great variability on the prevalence of CPEO and LHON among different ERR districts. This may probably be explained by different degrees of awareness about CPEO and LHON or by different neurological care approaches. Due to emerging new therapies for these diseases a policy to increase awareness about them is advised. Data obtained in the LHON of Bologna are similar to those previously observed in the North East of England as for LHON. Our data on $\mathrm{CPEO}$ are the first in the literature.

\section{9}

\section{Inter-Rater Agreement of the Romanian version of a Lifestyle Questionnaire}

\author{
A. Cucovici ${ }^{1,3,4}$, S. Arcuti ${ }^{2}$, M. Ferrara ${ }^{1}$, G. Chiumento ${ }^{1}$, N. Sirbu ${ }^{1}$, \\ V. Alexa ${ }^{3}$, A. Racovita ${ }^{3}$, M. Zarrelli ${ }^{1}$, V. Lisnic ${ }^{3}$, M.A. Leone ${ }^{1}$ \\ ${ }^{1}$ Neurology Unit and ${ }^{2}$ Unit of Biostatistics, IRCCS "Casa Sollievo \\ della Sofferenza", San Giovanni Rotondo, Italy; ${ }^{3}$ Institute of \\ Neurology and Neurosurgery, Chişinău, Republic of Moldova; \\ ${ }^{4}$ University of Foggia, Foggia, Italy
}

Objective: To evaluate the inter-rater agreement of the Romanian version of a life-style questionnaire used in an ongoing multicenter cross-sectional study on risk factors for Amyotrophic Lateral Sclerosis (ALS) progression. Background: Several life-style habits, including smoking, consumption of alcoholic beverages, coffee, tea, and foods containing antioxidants substances, have been evaluated as possible risk factors for ALS progression. We are currently doing a multicenter study in three countries (Italy, Moldova and Romania), using a validated questionnaire.

Methods: The Italian questionnaire was translated in Romanian by a Romanian mother language and back-translated in Italian by an Italian mother language. We assessed 40 patients admitted to the Institute of Neurology in Chisinau with different neurological disorders, who were interviewed by two trained raters, blinded to the interviewee's diagnosis and to each other's responses. The sequence of the interviews was randomized, the randomization list was concealed. Intraclass correlation coefficient was used to determine the level of agreement between the two raters for continuous variables, and Cohen's kappa statistics for nominal variables.
Results: Agreement was excellent (0.84 to 1$)$ for the broad indicators of current consumption (yes/no) for each type of alcoholic beverage and for age at drinking onset (0.997). However, when analyzing the current intake in grams/day, agreement was much better for aperitifs/spirits than red wine, white wine or beer, and only moderate (0.58), considering the total daily consumption of alcohol. An almost perfect agreement was obtained for age at smoking onset and quitting, patterns of smoking, current number of cigarettes, pack load, and passive smoking (0.94-1). Agreement was excellent (0.77-1) for current consumption of coffee, green tea, juices and foods containing antioxidants (0.84-1).

Conclusion: Although we obtained excellent agreement in most items of the questionnaire, we must acknowledge a lower agreement for the daily quantity of single beverages, and the total daily amount of alcohol intake. The inter-rater agreement of the Italian version of the same questionnaire was higher $(>0.90)$ for the yes/not questions for most beverages, but showed a trend similar to the Romanian version, being lower for the amount of intake of the single beverages.

10 Are Alcohol Consumption and Smoking Associated
with Multiple Sclerosis Disability?

A. Cucovici ${ }^{1,5}$, A. Ivashynka ${ }^{3}$, S. Arcuti ${ }^{1,2}$, D. Vecchio ${ }^{3}$, P. Naldi ${ }^{4}$, S. D'Alfonso ${ }^{3}$, M. Copetti ${ }^{2}$, M.A. Leone ${ }^{1}$

${ }^{1}$ Neurology, and ${ }^{2}$ Biostatistics Units, IRCCS "Casa Sollievo della Sofferenza", San Giovanni Rotondo, ${ }^{3}$ Department of Health Sciences, and ${ }^{4}$ SCDU Neurology, University of Piemonte Orientale, Novara, ${ }^{5}$ University of Foggia, Foggia, Italy

Objective: We aimed to evaluate the association of smoking and alcohol drinking with the clinical course and progression of Multiple Sclerosis (MS), using a cross-sectional study design.

Background: There is evidence that environmental exposures are important for the progression of MS, as well as for the risk of development of disease.

Methods: A sample of MS patients (McDonald criteria) was recruited in the Novara Hospital, a first-referral MS Center, during 2009-2013. Patients were enrolled during follow-up visits scheduled outside of relapse. Severity of MS was assessed through the Multiple Sclerosis Severity Score (MSSS). Smoking and alcohol history were evaluated with the European Prospective Investigation into Cancer and Nutrition (EPIC) lifestyle questionnaire. Interviews were made immediately after the visit and written informed consent, by two interviewers who were blinded as to clinical history and disability evaluation.

Results: Our sample included 351 consecutive patients, 190 ever-smokers and 283 ever-drinkers. Sex ratio (F/M) was 2.03:1 and relapsing-remitting form accounted for $76 \%$. Mean age at onset was $33.0 \pm 10.1$ years, median time from disease onset to MSSS evaluation was 10.0 (range: 1.0-48.0) years with a median MSSS of 2.70 (range: 0.1-9.9). Age- and gender-adjusted logistic regression models were estimated comparing the upper versus the lower tertile of the MSSS distribution. Ever-smokers (drinkers or non-drinkers) were almost 2 times more likely to fall in the upper tertile compared with never-smokers $(\mathrm{OR}=1.9,95 \%$ Confidence 
Interval $=1.1-3.3 ; \mathrm{p}=0.024)$. No dose-dependent associations were found between smoking or alcohol use and MS severity. The group of ever-smoker/never-drinker patients was six times more likely to fall in the upper MSSS tertile compared with the group of never-smoker/never-drinker patients $(\mathrm{OR}=6.4,95 \% \mathrm{CI}=1.5-$ 27.6; $\mathrm{p}=0.013)$. The OR to fall in the upper MSSS tertile was not statistically significant for the group of ever-smokers/ever-drinkers $(2.4 ; \mathrm{CI}=0.9-6.5)$ and of never-smokers/ever drinkers (1.6; $\mathrm{CI}=0.6-4.3)$ compared to never-smokers/never-drinkers.

Conclusion: Our study shows that smoking is associated with more severe disease and provides support to the hypothesis that alcohol consumption may attenuate this association. Incident patients recruited in this study are currently followed-up to evaluate the same possible predictors of progression with a prospective design.

\section{1 \\ The Diagnosis of Cognitive Disorders: The Population Persepctive from Normal to $\mathrm{MCl}$ and Dementia with Neuropathology as Gold Standard}

David A. Bennett

Rush Alzheimer's Disease Center, Chicago, IL, USA

Objective: To describe an actuarial decision tree approach to diagnoses of MCI and dementia in a longitudinal communitbased clinical-pathologic cohort study, with clinical-pathologic correlation.

Background: Community-based longitudinal studies are sensitive to diagnostic drift over time and space. Such drift introduces random variabililty at best, and bias at worst, for understanding risk factor associations with $\mathrm{MCI}$ and dementia.

Methods: More than 3,300 persons without known dementia enrolled in one of two community-based clinical-pathologic cohort studies, the Religoius Orders Study (ROS) or the Rush Memory and Aging Project (MAP). Enrollment began in 1994 and 1997 respectively. Clincial diagnoses are conducted each year blinded to previously collected data. Diagnosis of MCI and dementia, including Alzheimer's disease (AD) dementia, are informed by an actual decision tree that combines computer algorithm summaries of cognitive tests, and clinical judgement by a neuropsychologist, and clinician skilled at making dementia diagnoses. The approach was designed to be cost efficient, ensure that diagnoses did not drift over the multiple years and multiple clinicians, and efficiently reclassify all particpants in the event of updates to diagnostic criteria. Overall follow-up rate exceeds $90 \%$. The autopsy rate exceeds $80 \%$ with more than 1,500 autopsies to date.

Results: Using change point models, we show that incident MCI and incident AD dementia are associated with a markedly increased rate of cognitive decline. More than $99 \%$ of persons with a diagnosis of dementia met pathologic criteria for one or more pathologies, including nearly $85 \%$ with $\mathrm{AD}$, nearly all of whom had a co-existing pathology. Further, more than $95 \%$ of those with MCI met pathologic criteria for one or more pathologies., including $60 \%$ with $\mathrm{AD}$ nearly all of whom with co-existing pathology. Remarkably, more than $85 \%$ of persons without cognitive impair- ment met pathologic criteria for one or more pathologies, including more than $40 \%$ with $\mathrm{AD}$, most of whom had a co-existing pathology. In fact, there were nearly 250 unique combinations of brain pathologies with no single one present in more than $10 \%$ of persons. Further, the impact of any individual pathology on cognitive decline varied widely depending on the extent of co-morbid pathologies.

Conclusion: Cost efficient diagnoses of MCI and dementia can be made in community based longitudinal studies. However, the specificity of such diagnoses for specific causes of dementia is hampered by the multitude of mixed pathologies.

\section{2 \\ Estimating the Association of 5HTTLPR Polymorphism with Delusions in Alzheimer's Disease}

G. D'Onofrio, G. Paroni, D. Sancarlo, M. Lauriola, M.P. Dagostino, A. Mangiacotti, P. Bisceglia, C. Gravina, M. Urbano, F. Addante, F. Paris, L. Cascavilla, F. Panza, A. Greco, D. Seripa

Complex Structure of Geriatrics, Department of Medical Sciences, IRCCS “Casa Sollievo della Sofferenza”, San Giovanni Rotondo (FG), Italy

Objective: Serotoninergic pathways underlying delusion symptom in Alzheimer's disease (AD) have not been fully clarified. Aim of the present study was to investigate the association of 5-hydroxytriptamine (5-HT) transporter (5-HTT) gene-linked polymorphic region (5-HTTLPR) with delusions in patients with $\mathrm{AD}$.

Background: 5-HTTLPR is a variable number tandem repeats in the promoter region of serotonin transporter encoding-gene affecting transcription.

Methods: A total of 257 consecutive patients clinically diagnosed as $\mathrm{AD}$ according to the NIAAA criteria, were enrolled in the study and investigated for 5-HTTLPR. All participants underwent a comprehensive evaluation with the standardized Comprehensive Geriatric Assessment (CGA), Mini-Mental State Examination (MMSE), and Neuropsychiatric Inventory (NPI).

Results: Delusion symptom was observed 171 patients (66.54\%). In respect to $\mathrm{AD}$ w/o delusion, $\mathrm{AD}$ with delusion showed a low prevalence of S-plus carriers (5-HTTLPR-L/S+5HTTLPR-S/S genotypes) ( $\mathrm{p}<0.001$; OR $=0.240$, 95\%CI $0.121-$ $0.471)$. Logistic regression analysis adjusted for the apolipoprotein $\mathrm{E}$ (APOE) polymorphism showed that in $\mathrm{AD}$ with delusion the presence of an 5-HTTLPR-S allele may reduce disease duration $(\mathrm{p}=0.005 ; \mathrm{OR}=0.680,95 \%$ CI $0.522-0.886)$ and increase aberrant motor activity $(\mathrm{p}=0.013 ; \mathrm{OR}=2.257,95 \% \mathrm{CI} 1.195-4.260)$.

Conclusion: The present study suggested that 5-HTTLPR might be associated with delusions in AD. Whereas more studies on wide samples of high selected patients are needed to confirm our observation, the present results suggested important implication of serotonin regarding mechanisms underlying this symptom and his treatment. $\overline{80} \quad$ Neuroepidemiology 2018;50:74-103

DOI: $10.1159 / 000487692$ 15th Italian Congress of Neuroepidemiology, Tricase 2018 


\section{3}

\section{Assessing the Role of APOE Polymorphism on the Executive Dysfunctions in Alzheimer's Disease}

G. D'Onofrio, G. Paroni, D. Sancarlo, A. Mangiacotti,

P. Bisceglia, C. Gravina, M. Urbano, M. Lauriola, M. D'Agostino,

L. Cascavilla, F. Paris, F. Panza, A. Greco, D. Seripa

Complex Structure of Geriatrics, Department of Medical Sciences, IRCCS “Casa Sollievo della Sofferenza”, San Giovanni Rotondo (FG), Italy

Objective: To determine the role of the apolipoprotein $\mathrm{E}$ (APOE) $\varepsilon 4$ allele on executive dysfunctions in Alzheimer's disease (AD) patients.

Background: Up to date the APOE polymorphism is the major risk factors for sporadic $\mathrm{AD}$. No data regarding its role when executive dysfunctions are also present were available.

Methods: A total of $182 \mathrm{AD}$ patients attending Alzheimer's Evaluation Unit of Geriatrics, IRCCS Casa Sollievo della Sofferenza were included in the study. Of these patients, 132 were with executive dysfunctions (AD-ED) and 50 were without executive dysfunctions (AD-noED). All patients underwent APOE genotyping, Comprehensive Geriatric Assessment (CGA), Mini Mental State Examination (MMSE), Clinical Dementia Rating (CDR), Clock Drawing Test (CDT), and Frontal Assessment Battery (FAB).

Results: Percentage of females with AD-ED was significantly higher $(\mathrm{p}=0.044)$. AD-ED showed significantly higher cognitive impairment in MMSE $(\mathrm{p}<0.0001)$ and CDT $(\mathrm{p}=0.002)$, a more severe stage of dementia (CDR, $\mathrm{p}=0.008)$, and a worsening in several CGA domains (ADL, $\mathrm{p}=0.048$; IADL, $\mathrm{p}=0.015$; ESS, $\mathrm{p}=$ $0.035)$. A higher frequency of APOE $\varepsilon 4 / \varepsilon 4, \varepsilon 3 / \varepsilon 4$ and $\varepsilon 2 / \varepsilon 4$ genotypes was observed in patients with $\mathrm{AD}-\mathrm{ED}(82.4 \%, 97.6 \%$, and $66.7 \%$ respectively; $\mathrm{p}<0.0001)$. Conversely, a lower frequency of APOE $\varepsilon 3 / \varepsilon 3$ and $\varepsilon 2 / \varepsilon 2$ genotypes was observed in patients with $\mathrm{AD}-\mathrm{ED}(45.1 \%$ and $28.6 \%$ respectively; $\mathrm{p}<0.0001)$.

Conclusions: If confirmed in wider samples of subjects, the observed differences suggested that the presence of ED in $\mathrm{AD}$ might identify two clinically and diagnostically distinct groups of patients. The observed results may have important clinical implications regarding mechanisms underlying these symptoms that may implicate a different treatment of these patients.

\section{4}

Epi-Aortic Trunk Evaluation in Elderly Patients with and without Depression: A Case-Control Study

\author{
G. D'Onofrio' ${ }^{1}$, M.G. Longo ${ }^{1}$, M. Pacilli ${ }^{3}$, D. Sancarlo', \\ M.P. Dagostino' ', D. Seripa ${ }^{1}$, M. Lauriola' ${ }^{1}$, G. Paroni ${ }^{1}$, L. Cascavilla', \\ F. Paris ${ }^{1}$, F. Addante ${ }^{1}$, A. Mangiacotti ${ }^{1}$, S. Mastroianno ${ }^{3}$, \\ G. Di Stolfo ${ }^{3}$, V. Inchingolo ${ }^{2}$, M. Leone ${ }^{2}$, A. Russo ${ }^{3}$, A. Greco $^{1}$ \\ ${ }^{1}$ Complex Structure of Geriatrics, ${ }^{2}$ Complex Unit of Neurology, \\ Department of Medical Sciences, and ${ }^{3}$ Complex Unit of \\ Cardiology, Cardiovascular Department, IRCCS “Casa Sollievo \\ della Sofferenza", San Giovanni Rotondo, Foggia, Italy
}

Background: In older patients depression and atherosclerosis can occur. Their mutual relationships, however, are still unclear.

Objective: To investigate the occurrence of carotid atherosclerosis (CA) in patients with/without late-life depression (LLD/ noLLD), and the relationship between CA and LLD severity.

Methods: A total of 456 consecutive patients (333 LLD and 123 noLLD) attending the Ageing Evaluation Unit and Vascular Evaluation Unit of the IRCCS Casa Sollievo della Sofferenza were enrolled in the study. All patients underwent the standardized Comprehensive Geriatric Assessment (CGA), Mini-Mental State Examination (MMSE), Clock Drawing Test (CDT), Frontal Assessment Battery (FAB), Hamilton Depression Rating Scale (HDRS), and Carotid B-Mode Ultrasound scan.

Results: LLD patients showed significantly a higher grade of cognitive impairment (MMSE $\mathrm{p}=0.003$ ), a major impairment in any CGA domains (Activity of Daily Living, ADL $\mathrm{p}<0.0001$; Instrumental Activity of Daily Living, IADL $\mathrm{p}<0.0001$; Mini Nutritional Assessment, MNA $\mathrm{p}<0.0001$; Exton Smith Score, ESS: $p<0.0001$; social support network distribution: $p=0.017$ ), and more frequent white matter lesions (WMLs) $p<0.0001$ than noLLD. Very severe LLD patients had a higher grade of cognitive impairment (MMSE: $p=0.009$; FAB: $p=0.026$; CDT: $p=0.006$ ), and a major impairment in any CGA domains (ADL: $\mathrm{p}=0.006$; IADL: $\mathrm{p}=0.001$; MNA: $\mathrm{p}<0.0001$; ESS: $\mathrm{p}=0.003)$. WMLs were more frequent in Severe and Very severe LLD patients $(\mathrm{p}<$ $0.0001)$. The patients with atherosclerosis were mainly more depressed $(\mathrm{p}<0.0001)$, smokers $(\mathrm{p}=0.002)$ and with WMLs $(\mathrm{p}=$ 0.001 ) than patient without atherosclerosis. Patients with LLD demonstrated significantly a higher frequency in Moderate-severe atherosclerosis $(\mathrm{p}<0.0001)$. The severity of LLD seems increasing progressively in patients with Mild and Moderate-severe atherosclersis, showing that the patients with Very severe LLD were significantly more frequent in moderate-severe atherosclerosis $(\mathrm{p}=$ 0.002).

Conclusions: Subjects with atherosclerosis were more likely to be depressed. Moreover the severity of LLD seems increasing progressively in patients with Mild and Moderate-severe atherosclerosis. 
15

\section{Early Environmental Factors and Risk for Multiple Sclerosis: The Role of Breastfeeding}

Elisa Baldin ${ }^{1,2}$, Maura Pugliatti ${ }^{2}$, Trond Riise ${ }^{3}$, Anne-Kjestri Daltveit ${ }^{3}$

${ }^{1}$ Epidemiology and Biostatistics IRCCS Institute of Neurological Sciences of Bologna, Bologna, Italy; ${ }^{2}$ Department of Biomedical and Surgical Sciences, University of Ferrara, Ferrara Italy; ${ }^{3}$ Department of Global Public Health and Primary Care, University of Bergen, Norway

Objective: To assess the association between duration of exposure to breastfeeding and the occurrence of multiple sclerosis (MS) in offspring, by using prospectively collected populationbased data, and whether this association is modulated by a gender-specific role of breastfeeding.

Background: Early life exposure to environmental factors, such as nutrition, is hypothesized to be involved in the multifactorial pathogenesis of MS. Among these, breastfeeding has been reported in association with the development of immune-mediated diseases (inflammatory bowel disease, celiac disease, type-1 diabetes). Case-control studies suggest that prolonged breastfeeding may be associated with a decreased risk of MS both in childand adulthood.

Methods: Three datasets from Norwegian registries and surveys are linked for the study purpose: 1 . CONOR, a survey with mother-child records including information on breastfeeding, maternal health and lifestyle factors; 2 . Medical Birth Registry of Norway (MBRN): births in 1967-1999 with information on maternal and child health; 3. Norwegian MS-Registry (NMSR), with MS cases recorded up to $2016,60 \%$ coverage of the country and containing demographic and clinical information.

The national personal identification number is used to link the three datasets. The study population includes 122,286 children born in 1922-1999. For 85,198 of these born after 1967 there is complete information on pre- and perinatal factors. Duration of breastfeeding will be analyzed as continuous (months) and categorical (at least 3, 4, and 6 months).

Logistic regression will be used to estimate odds ratio (ORs) and $95 \%$ confidence intervals $(95 \% \mathrm{CI})$ to measure the association between MS and exposure to breastfeeding. All analyses will be adjusted for year of birth by 5-year categories and possible confounders (e.g. delivery mode, parity, gender, birth order) will be included.

Results: The median and mean duration of breastfeeding for the total study population was 6 and 6.4 months, respectively. Among these subjects 226 developed MS; in 207 cases (91.6\%) with a relapsing-remitting form, 15 (6.6\%) primary progressive, and $4(1.8 \%)$ unclassified. The median age at onset was 30 years (IQR: 14-55); the median age at diagnosis was 34 years (IQR: 17-71).

Conclusion: Breastfeeding as risk factor for MS has been evaluated by case-control studies. The use of prospectively collected national data gives the opportunity to evaluate possible associations, reducing the effect of bias and confounding factors.

\section{6}

\section{Long-Term Prognosis of Epilepsy: A Multi-Center} Retrospective Survey in Newly Diagnosed Patients

\author{
G. Giussani ${ }^{1}$, E. Bianchi', S. Beretta ${ }^{2}$, D. Carone ${ }^{2}$, C. Zanchi ${ }^{2}$, \\ M. Pirovano ${ }^{2}$, C. Trentini ${ }^{2}$, G. Padovano ${ }^{2}$, M. Colombo ${ }^{2}$, \\ D. Cereda ${ }^{2}$, S. Scanziani ${ }^{2}$, S. Gasparini ${ }^{3}$, G. Bogliun ${ }^{2}$, C. Ferrarese ${ }^{2}$, \\ E. Beghi ${ }^{1}$, The PRO-LONG Study Group \\ ${ }^{1}$ IRCCS Istituto di Ricerche Farmacologiche Mario Negri, Milano, \\ ${ }^{2}$ Epilepsy Center, Department of Neurology, San Gerardo \\ Hospital ASST Monza, University of Milano Bicocca, Monza, \\ ${ }^{3}$ Regional Epilepsy Center, Bianchi-Melacrino-Morelli Hospital, \\ Reggio Calabria, Italy
}

Objective: To predict the long-term outcome of newly diagnosed epilepsy after correct identification, classification and treatment; to identify prognostic indicators among selected demographic and clinical factors.

Background: Epilepsy is a chronic disease with a favorable response to the available treatments in the majority of patients. Studies in well-defined populations with newly diagnosed epilepsy have consistently shown a 1-year remission in up to $95 \%$ of people and up to $71 \%$ are in remission at last observation with or without drugs. Seizures in most people with epilepsy remit, but prognostic markers are poorly understood.

Methods: Multicenter retrospective, cohort study performed in 13 epilepsy Italian centers. Data were collected from clinical charts and, were needed, patients phone contacts. Patients were followed for a minimum period of 10 years. All remission periods were listed and classified in 1-year, 2-year, 5-year, and 10-year remissions based on the duration of seizure freedom for each period. The cumulative time-dependent probability of 1-year, 2-year, 5-year, and 10-year remission was calculated using KaplanMeier survival tables and curves.

Results: A total of 1006 children and adults were identified and followed for a cumulative period of 17,892 person-years. During follow-up, 923 patients (91.7\%) experienced $1+$ years of remission. 2-year, 5-year, and 10-year remissions were present in $89.5 \%$, $77.1 \%$ and $44.4 \%$ of cases. Seven hundred and 27 cases $(72.3 \%)$ were in remission at last follow-up. The cumulative probability of 1 -year remission was $66.4 \%$ at one year. The corresponding values at two, five, ten and twenty years were, respectively, $73.2 \%, 81.5 \%$, $87.6 \%$ e $92.1 \%$. At 20 years, the cumulative probability of 2 -year remission was $89.8 \%$. The corresponding probabilities were $79.2 \%$ for 5 -year remission and $46.4 \%$ for 10 -year remission. For each remission period, the variables associated with the highest chance of remission included a low number of seizures at diagnosis, a normal first imaging test, idiopathic generalized epilepsy, normal neurological and psychiatric signs, a low number of AEDs during follow-up, and no access to epilepsy surgery.

Conclusion: Our study provides robust evidence that more than $90 \%$ of patients with newly diagnosed epilepsy followed for more than 10 years, experience $1+$ years of remission, $79 \%$ has $5+$ years of remission, and almost one half of them has $10+$ years of complete seizure freedom.
Neuroepidemiology 2018;50:74-103

DOI: $10.1159 / 000487692$ 15th Italian Congress of Neuroepidemiology, Tricase 2018 


\section{7}

\section{Amyotrophic Lateral Sclerosis and Food Intake in Italy}

Elisabetta Pupillo ${ }^{1}$, Ettore Beghi ${ }^{1}$, Adriano Chiò ${ }^{2}$, Giancarlo Logroscino $0^{3,4}$

${ }^{1}$ IRCCS Istituto Mario negri, Milano, Italy; ${ }^{2}$ Rita Levi Montalcini ALS Center, Torino, Italy; ${ }^{3}$ Pia Fondazione Cardinale Panico, Tricase, Italy; ${ }^{4}$ Università degli Studi di Bari - Dip Scienze Mediche di Base, Neuroscienze ed Organi di Senso, Bari, Italy

Background: Many dietary factors have been investigated to verify their role in the onset or the progression of amyotrophic lateral sclerosis (ALS) without showing a clear beneficial or adverse effects. There are no studies focusing on specific nutrients.

Objective: To verify if specific (combinations of) foods and nutrients could be risk or protective factors in the development of ALS.

Methods: Patients with newly diagnosed ALS from 3 Italian administrative regions (Lombardy, Piedmont \& Valle D'Aosta, Puglia; total population 18.629.052) were included in a prospective observational study. Patients were included if they were aged $18+$ years and had definite, probable or possible ALS (El Escorial diagnostic classification). For each patient, a healthy control, matched for age ( \pm 5 years), sex and region of residence, was selected by a general practitioner. Cases and controls were interviewed and a validated and reproducible food-frequency questionnaire (FFQ) was filled. Daily intake of macronutrients, micronutrients, fatty acids, and total energy were estimated using an Italian food composition database.

Results: 212 cases and 212 matched controls were included (women, 188; men 236; mean age at diagnosis $62.6 \mathrm{yr}$; SD $10.4 \mathrm{yr}$ ). ALSFRS-R score at admission, 37.3 (SD 8.7). A risk reduction was found for coffee and tea $(\mathrm{OR}=0.29,95 \%$ CI $0.14-0.60$ for the highest versus the lowest quartile of consumption), whole bread $(\mathrm{OR}=0.55,95 \% \mathrm{CI} 0.31-0.99)$, raw vegetables $(\mathrm{OR}=0.25,95 \% \mathrm{CI}$ $0.13-0.52)$, citrus fruits ( $\mathrm{OR}=0.49,95 \%$ CI $0.25-0.97)$. A significant inverse association was observed for vegetable fat $(\mathrm{OR}=0.25$, $95 \%$ CI $0.11-0.57)$, total folate $(\mathrm{OR}=0.41,95 \% \mathrm{CI} 0.18-0.91)$, and vitamin $\mathrm{E}(\mathrm{OR}=0.40,95 \% \mathrm{CI} 0.18-0.92)$. After separating coffee and tea into subgroups, a significant risk reduction was observed for decaffeinated coffee $(\mathrm{OR}=0.18,95 \%$ CI $0.06-0.57)$ and tea ( $\mathrm{OR}=0.36,95 \%$ CI $0.19-0.67)$, but not for coffee with caffeine. A risk increase was observed for red meat $(\mathrm{OR}=2.96,95 \%$ CI $1.46-$ 5.99), pork and processed meat $(\mathrm{OR}=3.87,95 \% \mathrm{CI} 1.86-8.07)$, total protein $(\mathrm{OR}=2.96,95 \% \mathrm{CI} 1.08-8.10)$, animal protein $(\mathrm{OR}=$ 2.91, 95\% CI 1.33-6.38), sodium $(\mathrm{OR}=3.96,95 \%$ CI $1.45-10.84)$ and zinc $(\mathrm{OR}=2.78,95 \%$ CI 1.01-7.83).

Conclusion: Our findings support the hypothesis that some foods/nutrients may be risk factors and others protective factors for ALS. A larger and specific evaluation of few nutrients matched with specific genetic tests is awaited.

\section{8}

\section{How the Epidemiology of Epilepsy will Change After the New ILAE Definitions and Classifications of Seizures and Epilepsies?}

Ettore Beghi

IRCCS Istituto Mario Negri, Milano, Italy

Epilepsy is a clinical condition characterized by recurrent unprovoked seizures. For this reason, epidemiologists have always defined epilepsy as the occurrence of at least two unprovoked seizures. A new operational definition of epilepsy has been issued by the International League Against Epilepsy (ILAE) in 2014. According to this definition, epilepsy is a disease defined by any of the following conditions: 1 . At least two unprovoked (or reflex) seizures $>24$ hours apart; 2 . One unprovoked (or reflex) seizure and a probability for further seizures similar to the general recurrence risk (at least 60\%) after two unprovoked seizures, occurring over the next 10 years; 3. Diagnosis of an epilepsy syndrome. This revised definition implies that epilepsy can be also diagnosed after one unprovoked seizure, provided that factors are present suggesting a lowered seizure threshold. When applying the new definition of epilepsy, the incidence of the disease will increase significantly, and the increase in prevalence will be even greater. The prognosis of epilepsy and mortality will be also modified, making comparisons of disease trends virtually impossible. In 2017 the ILAE has also issued a new classification of seizures and epilepsies. The classification of seizures implies a basic definition of seizures as either focal, generalized or with unknown onset. Subcategories are then created for a more precise definition of old and new seizure types. According to the new classification of epilepsies, different diagnostic levels can be identified based on seizure type(s), epilepsy type and epilepsy syndrome. In contrast to the operational definition, with the new classifications of seizure and epilepsy types, significant differences are not expected in the distribution of broad seizure types because, at least in resourcepoor countries, the diagnosis may remain with basic categories. Differences in the distribution of seizure types and epilepsies may be, however, expected in resource-rich countries as a consequence of the use of sophisticated diagnostic aids (including genetic tests that attribute a genetic basis to several previously undefined cases). The forthcoming ICD-11 codes issued by the WHO have been aligned with the structure of the new classifications incorporating seizure-related codes, etiologies and epilepsy syndromes in the attempt to preserve the continuity with the previous codes (and related classifications). 


\section{9 \\ The Association Between Disulfiram and Parkinson's Disease}

F. D'Ovidio' ${ }^{1}$, A. d'Errico ${ }^{2}$, S.M. Goldman ${ }^{3,4}$, F. Ricceri ${ }^{5}$, P.D. Blanc ${ }^{3,4}$

${ }^{1}$ Department of Neuroscience, University of Turin, Turin, Italy;

${ }^{2}$ Department of Epidemiology, ALS TO3 Turin, Turin, Italy;

${ }^{3}$ Veterans Affairs Medical Center, San Francisco, USA; ${ }^{4}$ Division

of Occupational and Environmental Medicine, University of

California, San Francisco, USA; ${ }^{5}$ Department of Clinical and

Biological Sciences, University of Turin, Turin, Italy

Objective: To examine the association between exposure to disulfiram, a drug employed worldwide to treat alcohol use disorder (AUD), and the incidence of Parkinson's disease (PD).

Background: An increased risk of parkinsonism associated with exposure to carbon disulphide, particularly in rayon production, has been well documented. Disulfiram, which has carbon disulphide among its main metabolites, is still used as a therapeutic agent for AUD, but it is of concern that its use could increase the risk of PD or other parkinsonian syndromes.

Methods: The cohort study enrolled all inhabitants over 14 years of age in the city of Turin (a large Italian metropolitan area) who participated in the 1991 census and were still resident in Turin at the beginning of $1996(\mathrm{n}=681,283)$. Follow-up for PD started in 2002 and ended in 2012, while the exposure to disulfiram ranged between 1996 and 2012 (or 1 year before the first date of anti-parkinsonian drug prescription). Both disulfiram exposure and PD were assessed using the Regional ATC Drug Prescription Archive. Subjects with at least three prescriptions of disulfiram ("N07BB01") were considered exposed to this drug, while subjects with at least three prescriptions of any anti-parkinsonism drugs ("N04") were considered affected by PD. Exposure to disulfiram was treated as a time-dependent variable. A timespan dataset was constructed in order to run Cox proportional hazards models with robust standard errors, adjusted for sex, age in classes, educational level, and marital status.

Results: During the follow-up, 8,929 subjects developed PD (women: $52.8 \%)$, while $333(0.05 \%)$ were exposed to disulfiram (women: $30.9 \%$ ). Exposed cases were on average younger at PD medication initiation than those unexposed (50.8 vs. 72.9 years; Wilcoxon test $\mathrm{p}<0.001)$. The average time from initial disulfiram exposure to PD was 3.2 years $(S D=1.2)$. Fully adjusted Cox Proportional Hazard modeling indicated that disulfiram exposure was associated with the more than five-fold, statistically significant risk of PD (Hazard Ratio = 5.64; 95\% CI: 2.89-11.01).

Conclusion: This is the first population-based study demonstrating an increased risk of PD associated with disulfiram prescribing. The results suggest that disulfiram risk of PD should be taken into account in assessments of pharmaceutical safety in the treatment of AUD.

\section{0}

\section{Secular Trends of Amyotrophic Lateral Sclerosis.} The Piemonte and Valle d'Aosta Register

\author{
A. Chiò' ', G. Mora' ${ }^{2}$, C. Moglia' , U. Manera' , A. Canosa', \\ S. Cammarosano ${ }^{1}$, A.llardi ${ }^{1}$, D. Bertuzzo ${ }^{1}$, E. Bersano ${ }^{3}$, \\ P. Cugnasco' , M. Grassano' , F. Pisano ${ }^{4}$, L. Mazzini ${ }^{3}$, A. Calvo', \\ for the Piemonte and Valle d'Aosta Register for ALS (PARALS)
}

${ }^{1}$ Department of Neuroscience, University of Turin, Turin, Italy; ${ }^{2}$ ALS Center, "Salvatore Maugeri" Clinical-Scientific Institutes, Istituto di Ricovero e Cura a Carattere Scientifico, Milano, Italy; ${ }^{3} \mathrm{ALS}$ Center, the Azienda Ospedaliero Universitaria Maggiore della Carità, Novara, Italy; ${ }^{4}$ Department of Neurological Rehabilitation, "Salvatore Maugeri" Clinical-Scientific Institute, Istituto di Ricovero e Cura a Carattere Scientifico, Veruno, Italy

Objective: To examine the 20-year epidemiologic trends of ALS in the Piemonte and Valle d'Aosta regions of Italy.

Background: Although the epidemiology of ALS has been widely studied, in particular in Western countries, there are few data on the long-term epidemiologic trends of ALS.

Methods: The Piemonte and Valle d'Aosta Register for ALS (PARALS) is an epidemiologic prospective register that covers 2 Italian regions (population of 4,476,931 inhabitants according to the 2011 census) from January 1st, 1995, through December 31st, 2014. Case ascertainment is based on multiple sources (neurologic departments, hospital discharge archives, and mortality records). Incidence rates are age and sex standardized for the Italian population of the 2011 census. Age-period-cohort (APC) analysis was performed using a Poisson regression model.

Results: During the study period, a total of 2,702 patients (mean [SD] age at onset, 65.7 [11.1] years; 1,246 [46.1\%] female and 1,456 [53.9\%] male) received a diagnosis of ALS between 1995 and 2014, corresponding to a crude annual incidence rate of 3.03 per 100,000 population (95\% CI, 2.85-3.23) and an adjusted incidence rate of 2.78 per 100,000 population (95\% CI, 2.57-2.96). The age-adjusted incidence rate increased in the 2 decades of the study (1995-2004: 2.66; 95\% CI, 2.50-2.83; 2005-2014: 2.89; 95\% $\mathrm{CI}, 2.71-3.07 ; \mathrm{P}=0.04)$, mostly in women. The adjusted rate ratio of men to women decreased from 1.27:1 (1995-2004) to 1.17:1 (2005-2014). The analysis of deviance for the APC regression models indicated that the drift variable is relevant in explaining the variation of ALS incidence rates over time in the overall population (change in deviance, 4.6553; $\mathrm{P}=0.03$ ) and in women (change in deviance, 3.8821; $\mathrm{P}=0.05$ ) but not in men (change in deviance, $0.77215 ; \mathrm{P}=0.38$ ). A total of 479 patients with ALS were alive and had not undergone tracheostomy at the prevalence day (December 31st, 2014), corresponding to a crude prevalence rate of 10.54 per 100,000 population (95\% CI, 9.64-11.52).

Conclusion: During the 1995 to 2014 period, the crude and adjusted incidences of ALS increased in Piemonte and Valle d'Aosta, mostly in women. The APC model revealed that the increase of ALS incidence is attributable to a birth cohort effect in women, with a peak in the 1930 cohort. The different increase of ALS incidence in men and women points to an effect of exogenous factors with a differential effect on the 2 sexes, acting on a genetic background.
84

Neuroepidemiology 2018;50:74-103

DOI: $10.1159 / 000487692$ 15th Italian Congress of Neuroepidemiology, Tricase 2018 


\section{1 \\ Physical and Cognitive Frailty and Impact of Nutrition}

Francesco Panza 1,2,3, Madia Lozupone ${ }^{1}$, Rodolfo Sardone ${ }^{1}$, Vittorio Dibello ${ }^{1}$, Roberta Stallone ${ }^{1}$, Antonio Greco ${ }^{3}$, Antonio Daniele ${ }^{4}$, Davide Seripa ${ }^{3}$, Giancarlo Logroscino ${ }^{1,2}$

${ }^{1}$ Neurodegenerative Disease Unit, Department of Basic Medicine, Neuroscience, and Sense Organs, University of Bari Aldo Moro, Bari, Italy; ${ }^{2}$ Department of Clinical Research in Neurology, University of Bari Aldo Moro, "Pia Fondazione Cardinale G. Panico", Tricase, Lecce, Italy; ${ }^{3}$ Geriatric Unit \& Laboratory of Gerontology and Geriatrics, Department of Medical Sciences, IRCCS “Casa Sollievo della Sofferenza”, San Giovanni Rotondo, Foggia, Italy; ${ }^{4}$ Institute of Neurology, Catholic University of Sacred Heart, Rome, Italy

Frailty, a critical intermediate status of the aging process that is at increased risk for negative health-related events, includes physical, cognitive, and psychosocial domains or phenotypes. Clinical characteristics of frailty such as weakness, low energy, slow walking speed, low physical activity and weight loss underline the links between nutrition and frailty. In fact, poor nutritional status is one of the main risk factors for frailty. Macronutrients and micronutrients deficiencies are associated with frailty and recent studies suggested that improving nutritional status for macronutrients and micronutrients may reduce the risk of frailty. Specific diets such as the Mediterranean diet rich in antioxidants is currently investigated in the prevention of frailty. Cognitive frailty is another linked condition recently defined by operationalized criteria describing coexisting physical frailty and mild cognitive impairment (MCI), with two proposed subtypes: potentially reversible cognitive frailty (physical frailty/MCI) and reversible cognitive frailty (physical frailty/pre-MCI subjective cognitive decline). Several studies suggested a relevant heterogeneity with prevalence estimates ranging $1.0-22.0 \%$ (10.7-22.0\% in clinical-based settings and $1.0-4.4 \%$ in population-based settings). Cross-sectional and longitudinal population-based studies showed that different cognitive frailty models may be associated with increased risk of functional disability, worsened quality of life, hospitalization, mortality, incidence of dementia, vascular dementia, and neurocognitive disorders. The operationalization of clinical constructs based on cognitive impairment related to physical causes (physical frailty, motor function decline, or other physical factors) appears to be interesting for dementia secondary prevention given the increased risk for progression to dementia of these clinical entities. Multidomain interventions including also nutrition have the potential to be effective in preventing cognitive frailty. In the near future, we need to establish more reliable clinical and research criteria, using different operational definitions for frailty and cognitive impairment, and useful clinical, biological, and imaging markers to implement intervention programs targeted to improve frailty, so preventing also late-life cognitive disorders.

\section{2}

\section{Incidence of Neonatal Seizures and Perinatal Risk} Factors for Epilepsy and Mortality After Neonatal Seizures in the Province of Parma, Italy

\author{
Francesco Pisani ${ }^{1}$, Carlotta Facini ${ }^{1}$, Elisa Bianchi ${ }^{2}$, \\ Giorgia Giussani ${ }^{2}$, Benedetta Piccolo' ${ }^{1}$ Ettore Beghi ${ }^{2}$ \\ ${ }^{1}$ Child Neuropsychiatric Unit, Medicine and Surgery \\ Department, University of Parma, Parma, Italy; ${ }^{2}$ IRCCS-Istituto \\ di Ricerche Farmacologiche "Mario Negri", Milano, Italy
}

Objective: to evaluate the incidence of neonatal seizures confirmed by electroencephalogram and the perinatal risk factors for epilepsy and mortality after neonatal seizures. Background: there are few epidemiological data about neonatal seizures and they are mainly based on a clinical diagnosis of seizures.

Method: the neonates born in the province of Parma between January 2002 and December 2014 with electroencephalogramconfirmed seizures were identified and the incidence of neonatal seizures, for the entire sample and for sex, gestational age and birth weight was evaluated considering the number of babies born alive in Parma province in that period as reported in the EmiliaRomagna Certificate of Delivery Assistance data-base. Risk factors for mortality and, separately, for epilepsy after neonatal seizures were identified using univariate Cox's proportional hazards models. Risk factors that were statistically significant in univariate analysis were included in multivariate models. The study has been approved by the Institutional Review Board of the Parma University.

Results: the incidence of neonatal seizures was 2.29 per 1,000 live births (95\% CI, 1.87-2.72). The incidence was 2.38 per 1,000 (95\% CI, 1.78-2.99) in boys and 2.20 (95\% CI 1.61-2.81) in girls. The incidence in full-term newborns was 1.10 per 1,000 live births (95\% CI, $0.80-1.42$ ) and in preterm newborns was 14.28 per 1,000 live births (95\% CI, 10.78-17.79). Incidence was inversely correlated to gestational age and an increasing risk was also associated with decreasing birth weight. Among the perinatal risk factors that resulted significant in univariate models, only a birth weight $<2,500 \mathrm{~g}(\mathrm{p}=0.0154)$, a 5-minutes Apgar score between 0-3 ( $\mathrm{p}=$ 0.0062 ) and a severely abnormal electroencephalogram background activity $(\mathrm{p}=0.0154)$ retained significance in the multivariate analysis for mortality after neonatal seizures while the female gender (adj. HR 4.7, 95\% CI 1.5-14.8, $\mathrm{p}=0.0080$ ) and the neonatal status epilepticus (adj. HR 4.5, 95\% CI 1.4-14.1, $\mathrm{p}=0.0096$ ) for epilepsy.

Conclusions: Neonatal seizures are a fairly common clinical condition in the general population. The incidence of neonatal seizures is inversely associated with gestational age and weight at birth. A low birth weight, a low 5-minutes Apgar score and a severely abnormal electroencephalogram background activity are risk factors for mortality while the female gender and the status epilepticus for epilepsy after neonatal seizures. 
23

\section{Relationship Between Central and Peripheral Presbycusis and Mild Cognitive Impairment}

G. Logroscino ${ }^{1}$, R. Sardone ${ }^{2}$, M. Piccininni ${ }^{1}$, R. Tortelli, ${ }^{1,2}$, R. Capozzo 1,2, P. Mogavero 1,2, M.R. Barulli, ${ }^{1,2}$, A. Grasso ${ }^{2}$, M. Tursi $^{2}$, P. Battista ${ }^{1,2,4}$, C. Di Dio ${ }^{2}$, C. Griseta ${ }^{2}$, F. Coppola ${ }^{2}$, G. Sborgia ${ }^{2}$, G. Alessio ${ }^{2}$, N. Quaranta ${ }^{3}$,F. Panza ${ }^{1,2}$

${ }^{1}$ Department of Clinical Research in Neurology, University of Bari, “Pia Fondazione Cardinale G. Panico”, Lecce, Italy; Neurodegenerative Diseases Unit, Department of Basic Medical Science, Neuroscience and Sense Organs, University of Bari, Bari, Italy; ${ }^{2}$ RCCS "De Bellis" Castellana Grotte - The GreatAge Study; ${ }^{3}$ Otolaryngology Unit, University of Bari “Aldo Moro," Bari, Italy; ${ }^{4}$ Neurosciences, Psychology, Drug Research and Child Health (NEUROFARBA), University of Firenze, Firenze, Italy

Background: One third of elderly population has a disabling hearing loss worldwide and 5\% has a vision loss. Frailty is a multidimensional syndrome characterized by a general state of vulnerability, reduced physiological reserve, and increased susceptibility to common stressors. More than $20 \%$ of the elderly are frail.

Sensory impairments and frailty are both extremely common among the elderly and the prevalence of both increase with age Growing evidence supports an association between age-related hearing loss (presbycusis) and cognitive impairment, but few studies investigate the relationship between different types of presbicusys (peripheral and central), vision loss, frailty and presence of cognitive impairment.

Design/Methods: The Great-AGE Study is an on-going population-based study on aging, conducted in Castellana Grotte (Southern Italy). Subjects underwent multidisciplinary assessment including neurological, neuropsychological, geriatric and sense organs exams. Cognitive impairment was defined as MMSE lower than or equal 22, presence of peripheral and central presbycusis according to Gates (2012) criteria and visual acuity impairment as $\log$ MAR $>0.5$ according to WHO Factsheet of 2010. Frailty phenotype was defined by Fried 2001 criteria.

Results: In a sample of 574 individuals, who completed all the assessments, $21.1 \%$ had peripheral presbycusis and $8 \%$ had central presbycusis, $3.1 \%$ has vision loss. Presbycusis (OR $2.45 \mathrm{p}=0.002)$, central presbycusis $(4.46 \mathrm{p}=0.0002)$ and vision loss $(\mathrm{OR} 3.51 \mathrm{p}=$ 0.02 ) were associated with the presence of cognitive impairment, adjusting for age sex and education. No interactions was found between vision loss and presbycusis or central presbycusis. The prevalence of frailty was $11.9 \%$ and there was an association between frailty and cognitive impairment (OR $3.68 \mathrm{p}<0.001$ ).

Conclusions: In this elderly, Impairments in peripheral and central hearing, vision loss and frailty are associated to cognitive impairment in this elderly population. The findings suggest that frailty-related cognitive deficits may exist also depending on special sensory impairments.

\section{4}

Financial Capacity in Alzheimer Disease:

An Advanced Instrumental Activities or a Complex Multidimensional Neuropsycological Brain Function?

\author{
Piraino Giulia' , Caggiula Marcella ${ }^{2}$, Trianni Giorgio 3 , \\ Dell'Anna Andrea' ${ }^{1}$ Leo Antonio' \\ 'Presidio di riabilitazione funzionale "Istituto Santa Chiara" \\ Lecce, Italy; ${ }^{2}$ Scuola di specializzazione in Psicoterapia \\ cognitivo - comportamentale a Indirizzo Neuropsicologico; \\ ${ }^{3}$ Ospedale Vito Fazzi, Neurologia, Lecce, Italy
}

Objective: The aim of this work was to investigate and evaluate the specific cognitive skills involved in the functioning of FINANCIAL CAPACITY, using a new conceptual model able to take account of psychological and neurocognitive aspects and a new tool, the Questionnaire of Financial Capacity (QFC); first part of this tool aims at collecting general information and the second part assesses ten cognitive abilities of the person, applied in financial activities.

Background: One of the most common neurodegenerative processes in the human population is dementia: the degenerative process that characterizes it at the cognitive level has its behavioral counterpart in the loss of the daily autonomy and, consequently, of the patient's social role (Torralva, Dorrego, Sabe, Chemerinski, \& Starkstein, 2000). FINANCIAL CAPACITY (FC) represents one of the instrumental activities closely related to the daily autonomy of an individual. FC is a key capacity in everyday life, highlighting a list of clinical signs useful for the early assessment of this ability (Marson, et al., 2009; Marson D., 2001; Marson, et al., 2000). Depending on the primary importance for the autonomy and well-being of the elderly, clinicians must be careful to grasp the signs of a patient.

Methods: The sample included 28 subjects of both sexes and aged over 65 years; fourteen subjects with a neurodegenerative pathology and fourteen healthy subjects.

Results: The answers provided suggest a greater difficulty from the part of the EG compared to the CG. In general, the CG showed better performances than EG and specifically the shortcomings of women, both in the EG and in the CG. According to this the socio-cultural and the educational levels are related to the difficulties encountered. Behavioral and emotional reactions do not seem to be connected with the clinic condition.

Conclusion: The items of QFC were useful in the assessment of Financial Capacity, as they were able to describe the level of competence of each subject in the tasks assigned. The extension of the sample and the development of further analyzes to enhance the statistical validity of the tool will be the object of future research.
86

Neuroepidemiology 2018;50:74-103

DOI: $10.1159 / 000487692$ 15th Italian Congress of Neuroepidemiology,

Tricase 2018 
25

\section{Administrative Data and Rare Neurological Diseases}

G. Annicchiarico, G. Logroscino, E. Attolini, L.Bisceglia, V. Petrarolo, G. Gorgoni

Coordinamento Regionale Malattie Rare (CoReMaR) Agenzia Strategica Regionale per la Salute e il Sociale (AReSS) Puglia, Bari, Italy

Background: The Regional Rare Diseases Coordination (CoReMaR), of the Regional Strategic Agency for Health and Social in Puglia, has implemented since 2012 the Rare Diseases Information System of the Puglia Region. SIMaRRP is the logical infrastructure supporting the regional public assistance network. The Apulian network has developed with a logic of integration of the territory hospital. The SIMaRRP is fed by the diagnoses carried out in the National Network (PRN) Rare Diseases.

Methods: The analysis was conducted on the 12,250 patients included in the SIMaRRP as of 1 January 2017, thanks to the administrative data provided by EDOTTO (the Puglia health information system). For each patient the care path has been rebuilt through hospital admissions, specialized services in public or private affiliated facilities, local pharmaceutical consumption and attributable to File F.

Results: The prevalence estimate of the patients in their totality, and in particular of patients suffering from rare neurological diseases, in Puglia is superimposable to that of other regions. In 2016 more than two thirds of patients admitted to the study took place in Puglia. The cost of the overall assistance amounted to about 100 million euro, of which about 10 million were used for out-of-region care. The data are underestimated considering that currently they enjoy exemption from participation in the cost and the consequent rights due about 21,000 patients (EDOTTO) and that were considered as the only direct costs.

Conclusion: Population registers such as SIMaaRRP allow linking ICD9 codes with rare disease codes. Administrative data make it possible to reconstruct care pathways, mobility and costs in delayed times. The inclusion of PDTs (Therapeutic Diagnostic Plans) within the SIMaRRP will allow online monitoring of the assistance and the study of the reasons and appropriateness of the migration, considering that it is also evident the significant migration to non-accredited institutions such as PRN (diagnosis genetics, rehabilitation, etc).
26

\section{Borderline Personality Disorder and Type I Bipolar Disorder: An Integrated Cognitive- Neuropsychological Approach for a Better Clinical and Diagnostic Management}

Laura Miglietta ${ }^{1}$, Rosa Fanelli ${ }^{2}$, Antonio Leo ${ }^{3}$

${ }^{1}$ Scuola di Specializzazione in Psicoterapia CognitivoComportamentale a indirizzo Neuropsicologico, Lecce, ${ }^{2}$ Istituto Santa Chiara Lecce e Roma; Vicedirettore e docente Scuola di Specializzazione in Psicoterapia Cognitivo-Comportamentale a indirizzo Neuropsicologico, Lecce, ${ }^{3}$ Neurologo, Direttore Sanitario Presidio di Riabilitazione Funzionale "Istituto Santa Chiara" Lecce; Direttore Sanitario Centro Medico specialistico e di Riabilitazione "Istituto Santa Chiara" Roma; Vicedirettore e Docente Scuola di Specializzazione in Psicoterapia CognitivoNeuropsicologica Integrata "Istituto Santa Chiara" Roma, Italy

Objective: Inspection about how borderline personality disorder and bipolar type I disorder may change the performance during neuropsychological tests in the lack of emotional involvement, in tasks which need executive, attentive and memory functions involvement.

The adoption of a cognitive-neuropsychological approach for a better clinical and diagnostic management.

Background: About the $20 \%$ of patients affected by borderline disorder shows a comorbility for a bipolar disorder and about the $15 \%$ of patients with bipolar disorder has a borderline personality disorder. (Fiedorowicz J.G., Black D. W. 2010).

Through the study of the physiopathological basis which get involved in both disorders, it is noticed that they share the presence of emotional dysregulation. It involves control processes of the fronto-limbic network both at "top-down" and "bottom-up" levels (Malhi G.S., Tanious M., Fritz K. et al., 2003).

Methods: This study has been delivered by an integrated approach among neuropsychology, psychopathology and neurophysiopatology in order to have a more olistic vision of the patient's clinical and diagnostic management.

The attentive functions have been examined through trails of selective attention (Multiple Features Target Cancellation), division attention and cognitive flexibility (Trail Making Test). The memory functions have been inspected through verbal (Rey Auditory Verbal Learning Test, Prose Memory Test) and visual stimuli (Rey-Osterrieth Complex Figure). The executive functions have been evaluated through Tower of London Test (in order to evaluate the planning and problem solving ability) and Wisconsin Card Sorting Test (to assess the ability to shift cognitive strategies in response considering environmental contingencies).

Results: From the neuropsychological assessment is shown that the encoding phase and the cognitive flexibility are lower than standard limits.

The retrieval phase of non-semantic verbal linked items and visual stimulus are as substandard as planning ability.

Conclusion: In the lack of emotional involvement the patient shows substantial difficulties in the case of cognitive tasks which require planning, even behavioral; in case of flexible cognitive tasks, the patient's self-monitoring looks weak in the lack of external feedbacks.

Neuroepidemiology 2018;50:74-103 
The patient manages to inhibit her behavioral answers during a task purely cognitive and it appears difficult to her to use memory strategies and problem solving skills in absence of emotive cue.

This data can be useful for a more accurate cognitive-behavioral psychotherapy aimed to increase cognitive skills affected in both disorders.

27

\section{Administrative Data and the Future of Neurology}

Leandro Provinciali

Clinica Neurologica - Università Politecnica delle Marche, Ancona, Italy

Objective: To describe the relevant features of neurological care expected in the next years.

Background: Neurologic diseases are the leading cause of disability and death worldwide, so that a progress in the field of neurological care is stimulated. In coming decades a growing number of patients will need care by clinicians with expertise in neurological conditions, leading to an increase of professional resources and new models of neurology care.

Methods: A survey of new proposals for neurology care organization is provided focusing some aspect regarding neurologist careers.

Results: During the last years, some organization models were proposed to adapt the health organization to the increasing requests of neurological care. For example, a rapid access in the Emergency Department was able to prevent unnecessary admission and out-of doors neurology consultations, with high levels of patient satisfaction and cost savings (Ahmed et al. - MJA 192.1.2010). When the patients with neurological symptoms are evaluated in emergency department without a neurologist involvement, an uncorrect approach to patients presenting neurological symptoms decreases was revealed and incidence of correct management falls from 30 to 5\%, showing a high occurrence of misdiagnosis. (Pope \& Edlow - Emerg. Med Int. 2012).

A comprehensive approach to neurological disorders must improve the quality of care both in common diseases (dementia, stroke, headache, movement disorders, epilepsy and other parossistic conditions, neuropathy) and in low frequency neurological diseases.

The fundamental issues to be considered in planning the future of neurology may include new organization of neurological departments, more frequent clinical trials oriented to chronic disease, a striking communication of social importance of improvements in neurological care, as compared with cancer, cardiovascular and genetics (Martin \& Moses - Jama Neurol 2015).

A new organization of neurological services may prevent and mitigate neurologist burnout and promote engagement to satisfy the increasing requests (Miyasaki et al Neurology 2017).

Conclusion: Despite the observed decreases in mortality rates from stroke and communicable neurological disorders, the burden of neurological disorders has increased in the last years, because of the expanding aged populations. Policy makers and health-care managers should provide adequate services in order to save lifes and disabilities due to inappropriate neurological care.

\section{8}

\section{How to Assess the Quality of Clinical Practice Guidelines}

Luca Vignatelli

IRCCS Istituto delle Scienze Neurologiche di Bologna, Bologna, Italy

Clinical practice guidelines (CPG) "are statements that include recommendations intended to optimize patient care that are informed by a systematic review of evidence and an assessment of the benefits and harms of alternative care options" (Institute of Medicine 2011). Trustworthiness is the most important attribute of CPG. To be deemed trustworthy CPG should: be based on a systematic review; have been developed by a multidisciplinary panel of experts and representatives from key affected groups; be based on an explicit and transparent process that minimizes biases and conflicts of interest; provide a clear explanation of the logical relationships between alternative care options and health outcomes and provide ratings of both the quality of evidence and the strength of the recommendations. Consistent evidence shows that quality standards of CPG still are far from an average good level. These flaws seem to affect predominantly CPG produced by specialty societies. It is common to find recommendations, produced by different entities, on the same clinical question showing different advice or strength.

Formal appraisal of the quality of CPG is required if the selection of the best CPG or few CPG on a single topic is needed or if formal comparison among several CPG on the same topic is required for scientific or institutional reports. Critical appraisal of a selection of CPG could be fulfilled in three steps: 1 ) check for the minimal requirements to consider a CPG; 2) full assessment of the methods used to develop the CPG with the AGREE II instrument; 3) comparative analysis of methodological quality using AGREE II results, and comparative analysis of content of recommendations.
88

Neuroepidemiology 2018;50:74-103

DOI: $10.1159 / 000487692$ 15th Italian Congress of Neuroepidemiology, Tricase 2018 


\section{9}

\section{Social Dysfunction in Older Age. The GreatAGE Study}

Madia Lozupone, Francesco Panza, Marco Piccininni, Massimiliano Copetti, Rodolfo Sardone, Eleonora Stella,

Francesca D'Urso, Angela Lamanna, Maria Rosaria Barulli, Alessandra Grasso, Rosanna Tortelli, Rosa Capozzo,

Francesco Coppola, Daniela Isabel Abbrescia, Antonello Bellomo, Gianluigi Giannelli, Nicola Quaranta, Davide Seripa, Giancarlo Logroscino

University of Bari, National Institute of Health IRCCS S.De

Bellis - Castellana Grotte (BA) - Great Age Group, Bari, Italy

Background: Most studies focused on only one measure of social dysfunction in older age, without proper validation and distinction across different dimensions including subjectivity, structural and functional aspects.

Objective: We sought to validate the Social Dysfunction Rating Scale (SDRS) and its factorial structure and to determine the association of SDRS with cognitive functions, global psychopathology, and social deprivation.

Methods: We determined objective and subjective psychometric properties of SDRS against the gold standard evaluation of social dysfunction in psychiatric disorders according to the Semistructured Clinical Diagnostic Interview for DSM-IV-TR Axis I Disorders. The SDRS was administered to 484 Italian communitydwelling elderly, recruited in the GreatAGE study, a populationbased study on aging conducted in Castellana Grotte.

Results: The SDRS showed a moderate accuracy with an optimal cut-off of 26 maximized with higher sensitivity $(0.74,95 \% \mathrm{CI}$ : $0.63-0.84)$ than specificity $(0.57,95 \%$ CI: $0.50-0.64)$. A five-factor structure was carried out and five dimensions of SDRS were identified (loneliness; social isolation; feeling of contribution; leisure activities; anxiety for the health). Education and global cognitive functions were inversely correlated to SDRS, while a direct association with global psychopathology, depression, and apathy was found.

Conclusion: In older age, the SDRS could be a valid instrument to capture size and quality of social dysfunction, both in subjects with psychiatric disorders and in normal subjects. The prevalence of SDRS scores was higher in subjects with current psychiatric disorders vs. lifelong, but SDRS detected $40 \%$ of social dysfunction in normal subjects also.

\section{0}

\section{Preliminary Comparison of All-Cause Mortality Between Participants and Non-Participants in the Great-AGE Study}

M. Piccininni ${ }^{1,2}$, R. Sardone $e^{3}$, T. Lozupone ${ }^{3}$, G. Logroscino $^{1,2}$

${ }^{1}$ Department Basic Medicine Neuroscience Sense Organs, University of Bari, Bari, Italy; ${ }^{2}$ Department Clinical Research in Neurology, Unit of Neurodegenerative Disease, University of Bari, Hosp Panico, Tricase, Italy; ${ }^{3} \mathrm{NI}$ Gastroenterology "de Bellis", Research Hospital, Castellana Grotte, BA, Italy

Objective: To study participation bias in the Great Age, a population-based study on aging.

Background: Representativeness of the study sample is a major problem in population-based studies involving elderly subjects, as they are highly prone to non-participation. Response Bias, occurs when subjects who join the study are different from those who do not. The sample could be made recruiting the healthiest people of the randomly selected sample. An appropriate variable for measuring the global health status of a population is the mortality rate.

Methods: The Great Age study is an ongoing observational study on aging conducted in Castellana Grotte (Puglia, Italy) starting in June in 2012. We defined "participants" all subjects who joined the study before 31st October 2016. Survivorship functions and the mortality rates of both participants and nonparticipants were compared. Two Cox $\mathrm{PH}$ models were run: one with the recruitment status as covariate and the other including also sex and age at the first invitation.

Results: From $09 / 09 / 2012$ to $31 / 10 / 2016$, subjects invited to join the Great Age Study were 2578, of which 54.00\% (1392) accepted and $46.00 \%$ (1186) did not. At the first invitation, nonparticipants were older $(78.63 \pm 7.94)$ than participants $(73.9 \pm$ 6.68 ; $\mathrm{p}<0.001)$ more likely to be women $(60.54 \% 718)$ of nonparticipants $(\mathrm{p}<0.001)$. Compared to non-participants, participants showed a better survivorship $(\mathrm{p}<0.001)$. The mortality rate in the non-participants group was 65.11 (CI 95\%: 55.33-76.13) per 1000 py, while in the participants group it was 21.98 (CI 95\%: $17.55-27.18)$ per 1000 py. The mortality rate ratio was 2.96 (CI 95\%: 2.27-3.86), while the age-sex adjusted ratio was 1.95 (CI 95\%: 1.63-2.34). According to the unadjusted Cox Model, the ratio between the hazard in the non-participants and participants groups was 3.22 (CI 95\%: 2.46-4.21); the adjusted Cox Model, instead, showed an Hazard Ratio of 2.26 (CI 95\%: 1.70-3.00).

Conclusion: The difference in mortality between participants and non-participants confirms the presence of a Response Bias: participants were healthier. About $33 \%$ of the crude mortality ratio can be explained by a different demographic composition of the two groups, while $67 \%$ is not explainable by demographics as sex or age. 


\section{1 \\ Discrepancies Between Epidemiological Data on Dementias and Citizens Information}

\section{Maria Congedo ${ }^{1}$, Alberto Donzelli ${ }^{2}$}

${ }^{1}$ ASUIUD, ${ }^{2}$ Fondazione Allineare Sanità e Salute, Milano, Italy

Objective: Information about dementias is subject to mass media recurrent claim of "epidemic" diffusion. Epidemiological data need to be diffused to contrast over-investigation and to prevent over-diagnosis.

Background: Defeating Alzheimer disease and other dementias is an international priority in health politics. Prevalence and incidence trends of dementias show decrease in last decades in western countries, with some exceptions (the Netherlands). Instead, mass media report an "epidemic" spread, increasing fear for cognitive impairment. Typically, young family members of dementia patients ask to be investigated to identify predictive or early conditions. New criteria for Alzheimer disease aim to diagnose prodromal and preclinic conditions by biomarkers dosage in cerebrospinal fluid and amyloid neuroimaging whose specificity and sensitivity are very questionable. Uncertainty on the diagnostic value of biomarkers and neuroimaging can drive to over-diagnosis and to identification of a supposed risk condition that is untreatable.

Methods: Epidemiological data can be diffused by easyto-read, reference based medical information directed to citizens and physicians who can discuss before prescribing exams, avoiding alarmism. Some numbers of the publication "Pillole" by Fondazione Allineare Sanità e Salute considered this topic.

Conclusion: This approach can contribute to a better understanding of the limits of exams in use, reduce anxiety in family members of dementia patients, redirect towards useful and concrete preventive actions: eg healthy eating and drinking, exercising, quitting smoking, avoiding unnecessary drugs, especially anticholinergics.

\section{2}

\section{Pharmacoepidemiology of Multiple Sclerosis} Treatment in Veneto Region: An Observational Study

\section{A. Marangi, G. Farina, V. Vicenzi, S. Forlivesi, M.D. Benedetti \\ UOC Neurologia B, AOUI Verona, Italy}

Objective: The aim of this observational study is to describe Multiple Sclerosis (MS) treatment strategies used in the Hub and Spoke MS network in three Veneto Region provinces, covering a population of 2,675,144 inhabitants

Background: Pharmacoepidemiology studies the use, effectiveness and adverse effects of drugs in large populations. Efficacy and effectiveness of disease-modifying drugs (DMDs) used in MS are difficult to evaluate in clinical practice due to the heterogeneous clinical course of the disease, the lack of head-to-head randomized clinical trials and the pharmaeconomics analyses biased by conflicts of interest.
Methods: Demographic information, clinical features, DMDs history, and MRI data of patients regularly followed in the period January 2013 to December 2017 in the MS Centers of Verona, Vicenza and Treviso were recorded into a specifically designed database by the referral MS neurologists. McDonald (2010 revision) diagnostic criteria and Lublin (2013 revision) disease course classification were used to define clinical features, Expanded Disability Status Scale (EDSS) (Kurtzke,1983) to score disability. Persistence in treatment at 12 and 24 months, adverse effects and efficacy on relapses and disease progression were analyzed. Statistical analyses were performed by using SPSS v. 21.

Results: Out of 2997 MS patients enrolled in the study $64 \%$ are currently in treatment with first (29\% injective and $18 \%$ azathioprine or new oral drugs) or second-line (17\%) treatments. Among 939 cases not in therapy $42 \%$ assumed at least one DMD in the past. Overall $13 \%$ of patients never assumed any therapy, $26 \%$ assumed one DMD, 37\% at least two, $17 \%$ three and $7 \%$ four or more. Among those people who stopped old first-line therapies there was no difference in lack of efficacy between interferons beta (IFNBs) (43\%), glatiramer acetate (GA) (45\%) and azathioprine (AZA) (41\%), while adverse effects were the reasons to stop in $44 \%$ of IFNBs, $36 \%$ of GA and $30 \%$ of AZA groups. Only $8 \%$ stopped natalizumab for lack of efficacy against $40 \%$ of cases in fingolimod and $22 \%$ of cases in dimethylfumarate or teriflunomide.

Conclusion: Cost-effectiveness analyses are appropriate for highly efficacious first or second-line MS treatments, while a costminimization analysis may be useful for those drugs with analogous profile of efficacy and adverse events.

This study was funded by a grant from Veneto Region (Italy) (RP21400000415 RSF).

\section{3}

Use of Psychotropic Drugs in a Wealthy Italian Region (2007-2011). A Population Based Study in Employed Adults

\author{
Giorgio Vittadini ${ }^{1}$, Massimiliano Beghi ${ }^{2}$, Gloria Ronzoni ${ }^{3}$, \\ Mario Mezzanzanica 4 , Cesare Maria Cornaggia ${ }^{4}$
}

${ }^{1}$ Department of Statistics, University of Milano Bicocca, Monza, Italy; ${ }^{2}$ Department of Mental Health, AUSL Romagna, Italy; ${ }^{3}$ CRISP, University of Milano Bicocca, Milan, Italy; ${ }^{4}$ School of Medicine and Surgery, University of Milano Bicocca, Monza, Italy

Background: Over years, there has been an increase in the prescription of psychotropic drugs (PDs), particularly antidepressants (ADs).

Objectives: The aim of the study was to evaluate the consumption of PDs in adult employees in a productive area of Italy and the possible changes induced by the "Great recession"

Methods: The study is a retrospective survey in all adult employees in Lombardy, Northern Italy, aged $>18$ years in the period 2007-2011, classified by gender, age class, nationality, education and province.

Results: During the 5-year period, there were 3,554,860 employed adults in Lombardy, of whom 277,865 (7.8\%) used PDs.
90

Neuroepidemiology 2018;50:74-103

DOI: $10.1159 / 000487692$ 15th Italian Congress of Neuroepidemiology, Tricase 2018 
The percentage of PD users progressively increased from $3.37 \%$ in 2007 to $4.08 \%$ in 2011). The annual rate of increase was $7 \%$ in $2008,5 \%$ in $2009,5 \%$ in 2010 , and $3 \%$ in $2011 ; 16.1 \%$ of the cases used PDs in each of the 5 years. In $49.4 \%$ of the patients, the use of PDs was limited to 1 year, and $38.7 \%$ received them in only one trimester. The increase is attributable to AD and to MS with Anxyolitic and analgesic (in particular in neuropathic pain) properties as Pregabalin and Gabapentin, while concerning the AP the number of subjects treated is similar. The seasonal trend in $\mathrm{AD}$ prescriptions (with peaks in the fall and winter) was the same in males and females (although the prevalence of females was almost twice that of males) (seasonal $\chi 2$ test32.36667, p-value <0.001). The increase involved all the provinces in Lombardy in a similar manner.

The logit analysis showed that the use of PDs (particularlyADs) was associated with being an Italian woman aged $>55$ years with a basic education, a blue collar job, and an unstable working position.

Conclusions: The increase in the number of prescriptions of PDs after the economic crisis was the same as before it. The increase in PD use can be attributed more to Ads and anti-epileptic drugs with anxiolytic properties.

\section{4 \\ The Diagnosis of Psychogenic Non Epileptic Seizures (PNES): Can Video Alone Predict the Diagnosis? Comparison Between Epileptologists and Psychiatrists}

\author{
Massimiliano Beghi ${ }^{1}$, Giuseppe Erba ${ }^{2}$, Cesare Maria Cornaggia ${ }^{3}$, \\ Adriana Magaudda ${ }^{4}$, Valentina Chiesa ${ }^{5}$, Angela Laganà ${ }^{4}$, \\ Gabriella Di Rosa ${ }^{4}$, Giorgia Giussani ${ }^{6}$, Elisa Bianchi ${ }^{6}$, Gianni Porro $^{7}$, \\ Michela Russo ${ }^{6}$, Ettore Beghi ${ }^{6}$. \\ ${ }^{1}$ Department of Mental Health, AUSL Romagna, Italy; \\ ${ }^{2}$ Department of Neurology, SEC, University of Rochester, \\ Rochester, NY, USA; ${ }^{3}$ School of Medicine and Surgery, \\ University of Milano Bicocca, Monza, Italy; ${ }^{4}$ Department of \\ Neuroscience, University of Messina, Italy; ${ }^{5}$ Neurology Unit 2, \\ San Paolo Hospital, Milan, Italy; ${ }^{6}$ Laboratory of Neurological \\ Disorders, "Mario Negri", Institute, Milan, Italy; ${ }^{7}$ Rehabilitation \\ Center "Corberi", San Gerardo Hospital, Monza, Italy
}

Background: At present, except for video-EEG monitoring (VEM), an instrument for the diagnosis of psychogenic nonepileptic seizures (PNES) is lacking.

Objective: To investigate if, when, and to what extent visual information contained in a video-recorded event allows epileptologists and psychiatrists to predict the diagnosis of PNES without the aid of electroencephalography (EEG) and to compare the accuracy and the criteria leading to the diagnosis by psychiatrists with those used by epileptologists.

Methods: Four neurologists practicing in epilepsy centers and four board-certified psychiatrists were asked to review 23 videos capturing representative events of 21 unselected consecutive patients admitted for long-term VEM. All raters were blind to EEG and clinical information. They were requested to (1) rate the videos for quality and content; (2) choose among four diagnoses: (a) epileptic seizures (ES); (b) PNES; (c) Other nonepileptic seizures (NES); (syncope, movement disorder, migraine, etc.); (d) "Cannot Say"; and (3) explain in their own words the main reasons leading to the diagnosis of choice.

Results: All psychiatrists were concordant and correct (4/4) in $3 / 23$ video-events, compared to $8 / 23$ among epileptologists. The superiority of the epileptologists as a group is also apparent when the concordance in accurately predicting the diagnosis for each individual video was $<4 / 4$. Kappa values confirm the discrepancy between the two groups. While overall concordance among the four epileptologists was 0.50 (0.66 for PNES, 0.48 for ES and 0.20 for other NES), it was 0.18 (0.21 for PNES, 0.29 for ES and -0.03 for other NES) among the four psychiatrists. However, individual psychiatrists were almost as accurate as epileptologists in predicting the correct diagnosis. The comments provided to justify the diagnosis of choice differed from neurologists, varied among raters, and reflected considerable attention to body language, beside body movements.

Conclusion: Psychiatrists, as a group, are less accurate than neurologists in diagnosing PNES but, as individuals, they are as accurate in making the correct diagnosis and more attuned to capture the subtleties of human behavior, or subjective experiences as the effects of hidden internal conflicts, and can contribute a new lexicon in defining PNES.

\section{5 \\ Prognostic Relevance of Cerebrospinal Fluid Neurofilament Light Chain Level in Multiple Sclerosis: A Retrospective Single-Center Study}

M. Zanoni, E. Poletto, M. Bongianni, M. Turatti, M. Calabrese, A. Marangi, F. Gobbin, S. Monaco, A. Gajofatto

Università degli Studi di Verona, Dipartimento di Neuroscienze, Biomedicina e Movimento, Sezione di Neurologia, Verona, Italy

Objective: To determine whether neurofilament light chain (NFL) level in the cerebrospinal fluid (CSF) could represent an independent prognostic factor in multiple sclerosis (MS).

Background: NFL is an investigational biomarker with possible prognostic/predictive utility in MS.

Methods: We retrieved all available CSF samples from patients with clinically isolated syndrome (CIS) or MS, collected up to August 2014 and stored at Verona University Hospital Neuropathology Laboratory. NFL concentration was assessed using a commercially available ELISA kit. Clinical, MRI, and other CSF data were obtained retrospectively from medical records. Univariate analysis, Kaplan-Meier survival analysis, logistic regression (LR) and Cox proportional hazard models were used to study the association between NFL level and clinical outcomes of interest, including relapse occurrence and disability progression after lumbar puncture (LP).

Results: We analyzed 111 CSF samples collected from 72 female and 39 male patients with CIS $(n=25)$ or MS $(n=86,72$ with relapsing-remitting and 14 with progressive course), according to $2010 \mathrm{McD}$ onald criteria. Mean age at LP was $36 \pm$ 10 years. We observed a significant increase in CSF NFL level in patients with $\geq 1$ enhancing lesion on brain MRI closest to LP 
(3718 ng/L vs. $1589 \mathrm{ng} / \mathrm{L} ; \mathrm{p}=0.009$ ), as well as in patients with $\geq 1$ relapse after LP (3954 ng/L vs. $1597 \mathrm{ng} / \mathrm{L} ; \mathrm{p}=0.001)$. In addition, patients with NfL level $>2211 \mathrm{ng} / \mathrm{L}$ (i.e. median of study population) had a significantly shorter time to relapse (median 8.2 years vs 11.6 years; $\mathrm{p}=0.048$ ). We found a significant correlation of NFL concentration with relapse rate after LP $(\mathrm{r}=0.344, \mathrm{p}<0.001)$ and with expanded disability status scale (EDSS) score at one year after LP $(r=0.289, p=0.038)$. In the LR model, including age, sex, and measures of clinical and MRI activity at time of LP, CSF NFL $>2211 \mathrm{ng} / \mathrm{L}$ was the only variable significantly associated with subsequent relapse $(\mathrm{OR}=5.6,95 \%$ $\mathrm{CI}=2.3-13.7)$, while none of the examined variables was associated with EDSS progression at last follow-up. Cox regression models yielded no significant results for all the examined variables in the analysis of both relapse occurrence and EDSS progression.

Conclusion: CSF NFL is a marker of axonal injury linked to measures of clinical and MRI activity in MS. Increased NFL level in CSF appears an independent predictor of relapse. Additionally, CSF expression of NFL could possibly correlate with EDSS score at 1 year after LP.

36

\section{History of Allergies and Risk for Adult MS in the} Italian population: The EnvIMS study

\begin{abstract}
M. Pugliatti ${ }^{1,7}$, F. Badin ${ }^{2}$, E. Baldin ${ }^{1,3}$, I. Casetta ${ }^{1}$, E. Granieri ${ }^{1}$, K.-M. Myhr ${ }^{4}$, T. Riise ${ }^{5}$, C. Wolfson ${ }^{6}$

${ }^{1}$ Department of Biomedical and Surgical Specialty Sciences, University of Ferrara, Italy; ${ }^{2}$ School of Medicine, University of Ferrara, Italy; ${ }^{3}$ IRCCS Institute of Neurological Sciences, Bologna, Italy; ${ }^{4}$ Department of Neurology, Haukeland University Hospital; Department of Clinical Medicine, University of Bergen, Bergen, Norway; ${ }^{5}$ Department of Global Health and Lifestyle Epidemiology, University of Bergen, Norway; ${ }^{6}$ Department of Epidemiology and Biostatistics and Occupational Health, McGill University; The Research Institute of the McGill University Health Centre, Montreal, Canada; ${ }^{7}$ Department of Medicine, Division of Neurology, Faculty of Medicine, McGill University, Montreal, Canada
\end{abstract}

Objective: We aimed to investigate the association between multiple sclerosis (MS) and allergic conditions in relation to age, sex, and type of allergen.

Background: In line with the 'hygiene hypothesis', an excessive protection against infectious agents in infancy can challenge the immune system abnormally and increase allergic (Th2) and autoimmune (Th1) disorders. Although the Th1/Th2 dichotomy is an oversimplification and likely affected by a Th17 cell response, Th2 response may protect against Th1 mediated diseases, such as multiple sclerosis (MS).

Methods: We benefitted from data collected by means of EnvIMS a large multinational case-control study ultimately aimed to harmonize the methodology in investigating past exposure to environmental factors and risk for developing MS among adults. By means of a postal questionnaire (EnvIMS-Q), information on past medical history was collected from MS cases and frequency matched controls on sex and age, which included data on history of allergy (HoA) to pollen, house dust, animal fur/dander, food by age classes (5-year periods from birth to age 30 years). Logistic regression was used to calculate crude odds ratio (OR) with $95 \%$ confidence interval (95\% CI); OR was adjusted for sex, current age, smoking habit and educational level.

Results: A total of 2040 Italian study participants (707 cases, 1333 controls) contributed to the analysis. For all allergens no association between HoA and developing MS was detected in the total population (adjOR $=1.09$; 95\% CI: $0.89,1.33$ ). The distribution of exposure to allergies varied significantly between men $(35.5 \%)$ and women $(44.6 \%)$. When stratifying by sex, a higher frequency of exposure to allergies with onset at $0-5$ years was reported among MS women as compared to controls (adjOR: 2.74, $95 \%$ CI: $1.21,6.24)$. A significant association between allergy to house dust (onset at 0-10 years) and risk for MS among women (adjOR: 3.00, 95\% CI: 1.29, 6.99). Fewer men with MS reported exposure to food allergies with onset at $0-5$ years than controls $(\mathrm{OR}=0.61,95 \%$ CI: 0.46, 0.80).

Conclusions: Past HoA was more frequent among women with MS than controls, suggesting a common pathogenetic pathway as contemplated with the hygiene hypothesis. A gender effect was reported for early life onset of respiratory allergens conferring risk in women, and food allergens conferring protection in men. As for the latter, a role of gut microbiota cannot be ruled out.

Acknowledgements: FISM 2007/R/2014 e 208/R/19.

\section{7 \\ Teen Age Exposure to Diet Patterns May Modulate the Risk for Adult MS in the Italian Population and Norwegian: The EnvIMS Study}

M. Pugliatti ${ }^{1,7}$, B. Gullotta ${ }^{2}$, E. Baldin ${ }^{1,3}$, I. Casetta ${ }^{1}$, E. Granieri ${ }^{1}$, K.-M. Myhr ${ }^{4}$, T. Riise ${ }^{5}$, C. Wolfson ${ }^{6}$

${ }^{1}$ Department of Biomedical and Surgical Specialty Sciences, University of Ferrara, Ferrara, Italy; ${ }^{2}$ School of Medicine, University of Ferrara, Ferrara, Italy; ${ }^{3}$ IRCCS Institute of Neurological Sciences, Bologna, Italy; ${ }^{4}$ Department of Neurology, Haukeland University Hospital; Department of Clinical Medicine, University of Bergen, Bergen, Norway; ${ }^{5}$ Department of Global Health and Lifestyle Epidemiology, University of Bergen, Bergen, Norway; ${ }^{6}$ Department of Epidemiology and Biostatistics and Occupational Health, McGill University; The Research Institute of the McGill University Health Centre, Montreal, Canada; ${ }^{7}$ Department of Medicine, Division of Neurology, Faculty of Medicine, McGill University, Montreal, Canada

Objective: To investigate the association between patterns of diet during teen-age (13-19 years) and risk for MS in an Italian and a Norwegian population.

Background: Early life nutrition has been hypothesized to modulate the risk for adult multiple sclerosis (MS), as one of the potential environmental factors interacting with genetic susceptibility in the disease pathogenesis. The literature is controversial
Neuroepidemiology 2018;50:74-103

DOI: $10.1159 / 000487692$ 15th Italian Congress of Neuroepidemiology, Tricase 2018 
on association between diet and risk for MS, and at different times it focused on separately investigating the role of different items (eg., saturated fats, red meat, fish, salt, milk products, smoked meat etc).

Methods: We benefitted from data collected within the EnvIMS multinational case-control study aimed to identify a common methodology for analytical studies on environmental exposures and risk for MS.

The frequency of consumption of different foods was collected among MS cases and matched controls through a 6-page postal questionnaire, EnvIMS-Q. Logistic regression was used to calculate crude odds ratio (OR) with 95\% confidence interval (95\% CI); OR was adjusted for sex and smoking habit.

Results: A total of 4522 Italian and Norwegian study participants (1565 cases, 2957 controls) contributed to the analysis. Among the Italian study participants, controls vs MS cases reported a significantly more frequent consumption at teen-age of the following food: fresh tuna fish (adjOR $=0.79,95 \% \mathrm{CI}$ : $0.64,0.97$ ), cod fish (adjOR $=0.67,95 \%$ CI: 0.54, 0.83), anchovies/sardines (adjOR $=0.81,95 \%$ CI: 0.66, 0.99), smoked cheese (adjOR $=0.79,95 \%$ CI: 0.63, 0.97). Similar trends were also observed for consumption of other types of fish as well as for dairy products. Wuerstel consumption was more frequent among MS cases (adjOR $=1.25,95 \% \mathrm{CI}: 1.02,1.54)$. As for the Norwegian study population, consuming white fish, mackerel, and herrings was reported as more frequent among controls: $\operatorname{adjOR}=0.78$ (95\% CI: 0.65, 0.95), adjOR $=0.80$ (95\% CI: 0.67, $0.95)$, and adjOR $=0.71$ (95\% CI: 0.60, 0.85), respectively. Meat consumption (including smoked meat) during teen-age did not show any association

Conclusions: The study supports the hypothesis that diet patterns may interact with age (adolescence) to modulate the risk for developing adult MS. In line with existing literature, a potential protective role from fish consumption is observed in both populations, independently from vitamin $\mathrm{D}$ content and possibly reflecting lifestyle diet (more variety) patterns.

Acknowledgements: FISM 2007/R/2014 e 208/R/19.
38

\section{The Role of Birth Order and Sibship Size in Modulating the Risk for Multiple Sclerosis (MS) in Two Populations (Norway and Italy): The EnvIMS Study} M. Pugliatti 1,7 , A. De Marchi ${ }^{2}$, E. Baldin
1,3
K.-M. I. Casetta ${ }^{1}$, E. Granieri ${ }^{1}$,

${ }^{1}$ Department of Biomedical and Surgical Specialty Sciences, University of Ferrara, Ferrara, Italy; ${ }^{2}$ School of Medicine, University of Ferrara, Ferrara, Italy; ${ }^{3}$ RCCS Institute of Neurological Sciences, Bologna, Italy; ${ }^{4}$ Department of Neurology, Haukeland University Hospital; Department of Clinical Medicine, University of Bergen, Bergen, Norway; ${ }^{5}$ Department of Global Health and Lifestyle Epidemiology, University of Bergen, Bergen, Norway; ${ }^{6}$ Department of Epidemiology and Biostatistics and Occupational Health, McGill University; The Research Institute of the McGill University Health Centre, Montreal, Canada; ${ }^{7}$ Department of Medicine, Division of Neurology, Faculty of Medicine, McGill University, Montreal, Canada

Objective: To disclose and characterize an association between exposure to infant sibs, sibship size and the risk for multiple sclerosis (MS) in Norwegian and Italian populations.

Background: An association between exposure to hygienic conditions in childhood and the risk for developing immunemediated disorders explains the 'hygiene hypothesis'. One such exposure are childhood infections. Birth order and sibship size are reported as markers of such infections at early age.

Methods: We benefitted from data collected within the EnvIMS multinational case-control study aimed to identify a common methodology for analytical studies on environmental exposures and risk for MS. The number of sibs and their birth order was collected among MS cases and matched controls through a 6-page postal questionnaire, EnvIMS-Q. Logistic regression was used to calculate crude and adjusted odds ratio (OR) with $95 \%$ confidence interval (95\% CI).

Results: A total of 4687 Italian and Norwegian study participants, 1648 cases and 3039 controls contributed to the analysis. Norwegian MS cases were found to have been highly significantly less exposed to infant sibs (interbirth interval of $<3$ years) with a crude $\mathrm{OR}=0.58,95 \% \mathrm{CI} 0.48,0.70$ ), and after adjusting for sex, age at study time, educational level, history of infectious mononucleosis, smoking habit, sun exposure and duration of breastfeeding (adjOR $=0.61,95 \%$ CI: $0.49,0.75)$. Such association was not significant for cases with younger sibs with interbirth of 2 to 6 years, nor with older sibs (any age difference). No association with birth order was observed in the Italian population (any age difference), as well as in relation to MS age at onset (median $=37$ years) for both populations. After adjusting for the age at study time and in the Norwegian population only, sibships of 2 and over were found to be associated with younger age at MS onset $(\mathrm{OR}=0.47$, 95\% CI: 0.25, 0.89, $\mathrm{p}=0.021$ ).

Conclusion: Having been exposed to infant sibs with interbirth interval of $<3$ years can have a role in reducing the risk for adult MS. This is in line with studies on the Australian population. Large sibsize is found in association with earlier MS onset. These evidences were detected in the Norwegian study population only, 
and may therefore be population-specific. Other factors in the Italian population, eg., helminthes infection or other childhood infections, may be more relevant than birth order in modulating the risk.

Acknowledgements: FISM 2007/R/2014 e 208/R/19.

\section{9}

\section{The Prevalence of the Frontal Lobe Syndrome in a Population-Based Sample of 65 Year Olds: Preliminary Results from the Great-Age Study}

P. Battista ${ }^{1-3}$, M. Piccininni ${ }^{3}$, M. Barulli $^{3}$, C. Griseta $^{1}$, A. Grasso $^{1}$, C. Di Dio ${ }^{1}$, R. Stallone ${ }^{1}$, R. Capozzo ${ }^{3}$, M. Lozupone ${ }^{1}$, R. Sardone ${ }^{1}$, R. Tortelli ${ }^{1,3}$, F. Panza ${ }^{1,3}$, G. Logroscino ${ }^{1,3}$

${ }^{1}$ Department of Basic Medicine, Neuroscience, and Sense Organs, University of Bari Aldo Moro, Bari, Italy; ${ }^{2}$ Neurosciences, Psychology, Drug Research and Child Health (NEUROFARBA), University of Firenze, Firenze, Italy; ${ }^{3}$ Department of Clinical Research in Neurology, Neurodegenerative Diseases Unit, "Pia Fondazione Card G Panico"Hospital, Tricase, Lecce, Italy

Objective: To estimate the prevalence and phenotypic characteristics of frontal lobe syndrome (FLS) among the elderly (>65 y) in a population based study.

Background: The FLS can be characterized by a wide spectrum of symptoms grouped in three domains: executive functions, behavioral and social cognition (i.e., empathy and theory of mind deficits). A FLS is reported in numerous neurobehavioral conditions such as Frontotemporal lobar degeneration and other dementias, including Alzheimer's disease.

Methods: The Great-Age Study is an ongoing populationbased study on aging, conducted in Castellana Grotte (Southern Italy). Subjects underwent multidisciplinary assessment. In particular, neuropsychological examination included the Frontal Behavior Inventory (FBI) for detecting behavioral symptoms, and standardized tests for executive functions and social cognition. The FLS was defined using two algorithms: 1-broad (presence of dysfunction in at least 1 domain); 2-narrow (presence of behavioral symptoms - FBI $>11$ - PLUS executive dysfunction OR social cognition deficits).

Results: We evaluated a sample of 112 subjects ( 57 males, 55 females, mean age of $70.52 \pm 4.89 ; 8.27 \pm 3.79$ mean years of school education). The FLS was present in the $36 \%$ of subjects using the broad algorithm and in the $7 \%$ using the narrow algorithm. Considering the single components $8 \%$ presented a behavioral dysfunction, $14 \%$ social cognition deficit and $36 \%$ at least one deficit of executive functions.

Conclusion: These preliminary results showed that the FLS, if investigated in all its components, is quite frequent phenotype among the elderly.

Acknowledgment: This study was supported by AIRAlzhOnlus-COOP Italia to P. Battista and realized with the financial support of the Puglia Region.

\section{0}

\section{M.I.N.O.RE Project (Non-Compulsory Water Monitoring at Regional Level): Study Protocol}

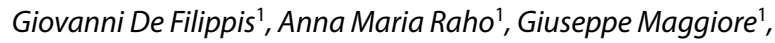 \\ Rizzo Emanuele ${ }^{2}$, Antonella De Donno ${ }^{3}$, Alberto Fedele 1 , \\ Roberto Carlà', Daniele Tondo', Sergio De Pascali' ${ }^{1}$, \\ Prisco Piscitelli ${ }^{2}$ \\ ${ }^{1}$ Local Health Authority of Lecce - Department of Prevention, \\ Lecce, Italy; ${ }^{2}$ Euro Mediterranean Scientific Biomedical Institute \\ (ISBEM), Mesagne, Italy, ${ }^{3}$ University of Salento - Department of \\ Biological and Environmental Sciences and Technologies \\ (DISTEBA), Lecce, Italy
}

Objective: This research origins from the will to evaluate the Salentinian groundwater quality: the whole area uptakes all the water needed from the local deep aquifer. A detailed study It's very interesting and urgent also considering the karst geology of the local subsoil, exposing deep water to surface pollutants.

Background: Recent epidemiological data from the Cancer Registry of Lecce, ISTISAN Reports and the Regional Epidemiological Observatory show an increasing incidence and mortality rate for tumors (in particular lung and bladder cancers), with a multifactorial eziology to which may also contribute foodand water-borne chemicals. Moreover, the ISPRA 2016 Report (on 2013-14 data) on groundwater quality in the various Italian regions stated that there was a lack of information for Apulia region, since the monitoring network for groundwater in Apulia was founded in 2015 with the Maggiore Project.

Methods: The methodology to follow will be based on six points:

1) Quali-quantitative extension of the monitoring of water intended for human consumption (increasing the total number of samples and analytes searched in wells managed by AQP and water in distribution);

2) Insights on food matrices and water for irrigation and breeding;

3) Integrated Assessment of Health Risk in Groundwater (V.I.R.S.A);

4) Epidemiological surveillance activity (Surveillance on workers exposed to known carcinogens and possible case-control study on bladder cancer);

5) Update of the first Environment and Health Report in the Province of Lecce;

6) Actions to inform the population about the correct use of water resources and healthy eating.

Expected Results: The data obtained from the analysis will contribute to improving the knowledge of the current state of health of the Salentinian aquifers, allowing also an insight on possible correlations between pollutant compounds and professional exposures, as well as specific assessments dealing with the prevalence of neurodegenerative disorders in the areas where pesticides will be found in the water.

Conclusion: The analysis of deep water quality in Salento, combined with epidemiological investigation on urothelial tumors and possible links between phytosanitary products and neurodegenerative diseases, will help to update the data produced by RePOL (Network for the Cancer Prevention of Lecce),
Neuroepidemiology 2018;50:74-103

DOI: $10.1159 / 000487692$ 15th Italian Congress of Neuroepidemiology, Tricase 2018 
after the presentation on February 16th 2016 of the first Report Environment and Health and the recent conclusion of the PROTOS case-control study on lung tumors.

\section{1 \\ Diagnosis and Clinical Course of Patients Presenting with an Inflammatory Demyelinating Event: Preliminary Results of an Ongoing Prospective Multicentre Study \\ S. Mariotto ${ }^{1}$, R. Orlandi ${ }^{1}$, S. Ferrari ${ }^{1}$, M. Turatti ${ }^{1}$, F. Gobbin ${ }^{1}$, A. Marangi ${ }^{1}$, D. Alberti ${ }^{1}$, F. Alessandrini ${ }^{1}$, A. Bonora ${ }^{1}$, F. Rossi ${ }^{3}$, M. Zoccarato ${ }^{6}$, L. Zuliani ${ }^{7}$, R. Bombardi ${ }^{8}$, C. Zuliani ${ }^{9}$, D. Ferraro $^{10}$, R. Capra ${ }^{11}$, C. Mancinelli ${ }^{11}$, S. Monaco ${ }^{1}$, M. Reindl ${ }^{12}$, A. Gajofatto ${ }^{1}$ \\ ${ }^{1}$ Verona, Italy; ${ }^{2}$ Peschiera del Garda, Italy; ${ }^{3}$ Legnago, Italy; ${ }^{4}$ Negrar, Italy; ${ }^{5}$ Trieste, Italy; ${ }^{6}$ Padova, Italy; ${ }^{7}$ Treviso, Italy; ${ }^{8}$ Bassano del Grappa (VI), Italy; ${ }^{9}$ Mirano (VE), Italy; ${ }^{10}$ Modena, Italy; ${ }^{11}$ Montichiari (BS), Italy; ${ }^{12}$ Innsbruck, Austria}

Objective: 1) To define the diagnosis and clinical course of patients with an initial demyelinating event (IDE) not fulfilling 2010 McDonald criteria for multiple sclerosis (MS); 2) To investigate the diagnostic and prognostic value of serum myelin oligodendrocyte glycoprotein antibodies (MOG-IgG) testing in such cases.

Background: IDEs may be related to various diseases, particularly MS and neuromyelitis optica spectrum disorder (NMOSD). However, the diagnosis is often delayed or undetermined, even after appropriate clinical and laboratory assessment. In this context, the significance of MOG-IgG testing compared to standard work-up is still uncertain.

Methods: Adult patients with an IDE not fulfilling 2010 McDonald criteria for MS in the 12 months prior to baseline visit are being enrolled in 7 Italian centres. Recruitment duration is 18 months; each patient is followed prospectively for at least 2 years. Baseline evaluation includes clinical assessment, brain and spinal cord MRI, cerebrospinal fluid (CSF) analysis, evoked potentials and blood work-up with MOG-IgG testing (live cell assay). Study assessments are repeated every 6 or 12 months and in case of relapse. At last follow-up visit patients are subdivided into distinct categories according to their diagnosis.

Results: By December 2017, 36 patients (23 females) have been enrolled. Median onset age was 43 (22-76) years. Clinical presentation was acute myelitis in 18 cases, optic neuritis in 9, brainstem/ cerebellum syndrome in 4 , cerebral syndrome in 2 , and multifocal in 3. Median EDSS score at baseline was $2(0-6) .6$ patients had a relapse during follow-up (median duration 6 [0-12] months). Diagnosis at last visit was clinically isolated syndrome (CIS) or MS according to 2010 McDonald criteria in $11+5$ patients, NMOSD in 5, and under investigation in 15 . We found 4 MOGIgG positive cases (one in the NMOSD group and 3 under investigation), characterized by the following, in comparison with MOG-IgG (and NMO-IgG) negative patients:

Conclusion: CIS and MS represent the most likely diagnosis after an IDE, even in patients not fulfilling $2010 \mathrm{McD}$ onald criteria at presentation. Our results suggest that MOG-IgG positive cases are around $11 \%$ in this population and they could possibly have distinct features compared to MOG-IgG and NMO-IgG negative patients. Confirmation on a larger sample is warranted.

\section{Relationship Between Central and Peripheral Presbycusis and $\mathrm{MCl}$ in a Population-Based Study of Southern Italy: The "Great Age Study"}

Background: One third of elderly population has a disabling hearing loss worldwide. Growing evidence supports an association between age-related hearing loss (presbycusis) and cognitive decline, but few studies investigate the relationship between different types of presbicusys (peripheral and central) and presence of MCI. No previous study has investigated speech discrimination and separate the auditory perception from auditory function.

Design/Methods: The Great-AGE Study is an on-going population-based study on aging, conducted in Castellana Grotte (Southern Italy). Subjects underwent multidisciplinary assessment including neurological, neuropsychological, geriatric and otolaryngology assessments. Mild cognitive impairment (MCI) was diagnosed according to Petersen criteria and presence of peripheral and central presbycusis according to Gates (2012) criteria.

Results: In a sample of 1604 individuals, $25.5 \%$ has peripheral presbycusis and $12.1 \%$, has central presbycusis Central presbycusis was associated with the presence of MCI (OR 2.1, p < 0.0001). In contrast, there was no association between peripheral presbycusis and cognitive impairment. Furthermore, there was a positive correlation between the average speech discrimination score and the MMSE score $(\mathrm{r}=0.53 \mathrm{p}<0.0001)$.

Conclusions: Our preliminary results suggest that central presbycusis may share with cognitive decline the same pathophysiological mechanism of neurodegeneration instead of being linked to a process of sensory deprivation given by peripheral presbycusis. Hearing perception tests should be included in the evaluation of persons older than 65 years and in those with cognitive impairment. 


\section{3 \\ The Incidence of FTLD in Salento: Preliminary Results from Puglia FTD Registry}

R. Capozzo ${ }^{1}$, R. Tortelli ${ }^{1}$, M. Piccininni ${ }^{1}$, P. Battista ${ }^{1-3}$, R. Sambati ${ }^{4}$, R. Barone ${ }^{1}$, C. Zecca ${ }^{1}$, G. Logroscino ${ }^{1,2}$

${ }^{1}$ Department Clinical Research in Neurology, Unit of Neurodegenerative Disease, University Bari, Hospital Panico, Tricase, Italy; ${ }^{2}$ Department Basic Medicine Neuroscience Sense Organs, University Bari, Bari, Italy; ${ }^{3}$ Department Neuroscienze psicologia area del farmaco e salute del bambino, University Firenze, Firenze, Italy; ${ }^{4}$ SSL Lecce, Italy

Objective: To estimate the incidence of the fronto-temporallobar degeneration (FTLD) in Puglia and in particular in the province of Lecce.

Background: FTLD is an umbrella term indicating a progressive degeneration of fronto-temporal lobes with an extreme clinical pathological and genetic heterogeneity. Epidemiology is based on few studies with adequate methodology.

Method: A registry for the study of epidemiology and genetics of FTLD has been built up in Puglia. FTD cases were enrolled according to the new diagnostic criteria for different clinical variants. We considered the behavioural variant of FTD (bvFTD) and the Language variants: Nonfluent Primary Progressive Aphasia (nfvPPA), Semantic Dementia (SD) and Other Language and Speech Disorders (OLSD). The spectrum was considered as inclusive of the motor phenotypes involving the primary motor pathways (Frontotemporal Dementia-Amiotrophycal Lateral Sclerosis FTD-ALS) and the extrapyramidal pathways (Progressive Sopranuclear Palsy PSP and Cortico Basal Degeneration CBD). The registry is based on a multisource referral system based both on memory clinics in the area and neurologists and geriatricians working in the local outpatient neurological clinics of the National Health System. The data are preliminary in an area of the registry (Province of Lecce inhabitants: 802082). Age, sex and age-sex specific incidence rates were computed. All rates were calculated using the population of the Province of Lecce of 01/01/2017 as reference (assuming no variation occurred in the 12 month of the study period).

Results: 28 cases of FTLD-associated syndromes were identified in the area over 12 months (01/01/2017-31/12/2017); 16 patients $(57.14 \%)$ were males and $12(42.86 \%)$ were females; 14 (50.00\%) were bvFTD, 4 (14.29\%) were nfvPPA and $5(17.86 \%)$ were PSP, 2 (7.14\%) were CBD, 3 (10.71\%) were OLSD. The mean age at diagnosis was $69.39 \mathrm{y}$. Incidence Rate for FTLD-associated syndromes was $4.17 / 100,000(2.38-6.77)$ py for males, $2.87 / 100,000(1.48-5.01)$ py for females and 3.49/100,000 (2.32$5.05)$ py for the general population. The Incidence curve by age at diagnosis category in general population showed a peak in the 65-69 class with an incidence of 17.49/100,000 (8.00-33.21) py.

Conclusions: These are the preliminary results of a new registry on FTLD: the incidence rates are higher than previously reported with predominance of male cases. The peak of incidence is shifted toward an older age groups compared to the majority of previous reports.

\section{4}

\section{Pseudobulbar Affect as a Negative Prognostic Indicator in Amyotrophic Lateral Sclerosis}

\author{
R. Tortelli ${ }^{1}$, S. Arcuti ${ }^{2}$, M. Copetti ${ }^{2}$, R. Barone ${ }^{1}$, C. Zecca ${ }^{1}$, \\ R. Capozzo ${ }^{1}$, M.R. Barulli', I.L. Simone ${ }^{3}$, G. Logroscino ${ }^{1,3}$
}

${ }^{1}$ Unit of Neurodegenerative Diseases, Department of Clinical Research in Neurology, University of Bari "A. Moro" at Pia Fondazione Card. G. Panico, Tricase, Lecce, Italy; ${ }^{2}$ Unit of Biostatistics, IRCCS "Casa Sollievo della Sofferenza", San Giovanni Rotondo, Foggia, Italy; ${ }^{3}$ Department of Basic Medical Sciences, Neurosciences and Sense Organs, University of Bari "A. Moro", Bari, Italy

Objective: To evaluate if presence of pseudobulbar affect (PBA) in an early stage of the disease influences survival in a population-based incident cohort of amyotrophic lateral sclerosis (ALS).

Background: PBA is a frequent symptom in ALS (up to $50 \%$ of cases over the whole course of the disease), often under-recognized and undertreated. In a previous study PBA has been related to a more severe ALS phenotype at diagnosis. To date there are no studies that evaluate the role of PBA as prognostic indicator in ALS.

Methods: Incident ALS cases, diagnosed according to El Escorial criteria were enrolled from a prospective population based registry in Puglia, Southern Italy. The Center for Neurologic Study-Lability Scale (CNS-LS), a self-administered questionnaire, was used to evaluate PBA. Total scores range from 7 to 35 . A score $\geq 13$ was used to identify PBA. Cox proportional hazard models were used for survival analysis. The modified C-statistic for censored survival data was used for models' discrimination. RECursive Partitioning and AMalgamation (RECPAM) analysis was used to identify subgroups of patients with different patterns of risk, depending on baseline characteristics.

Results: We enrolled 94 sporadic ALS, median age of 64 years (range: 26-80). At the censoring date, 65/94 (69.2\%), 39/60 $(65.0 \%)$ and $26 / 34(76.5 \%)$ patients reached the outcome (tracheotomy/death), in the whole, non-PBA and in the PBA group respectively. Kaplan-Mejer survival curves for the two subgroups were not significantly different (log-rank test: $1.3, \mathrm{p}=0.25)$. The discrimination ability of a multivariable model with demographic and clinical variables of interest was not improved by adding PBA. In the RECPAM analysis ALSFRSr and the total score of CNS-LS scale $(</ \geq 10)$ were the most important variables for differentiating all risk categories.

Conclusions: These preliminary results underlie that presence of PBA at entry negatively influences survival in a specific subgroup of patients with ALS characterized by less functional impairment. $\overline{96} \quad$ Neuroepidemiology 2018;50:74-103

DOI: $10.1159 / 000487692$ 15th Italian Congress of Neuroepidemiology, Tricase 2018 


\section{5}

Dementia and Vascular Risk Scores in an Aging Population: An Association with Cognitive and Sensory Impairment

R. Tortelli ${ }^{1}$, M. Piccininni ${ }^{1}$, P. Battista ${ }^{1}$, L. Di Lena ${ }^{2}$, D.I. Abbrescia ${ }^{2}$, M.R. Barulli' , R. Capozzo ${ }^{1}$, F. Coppola ${ }^{2}$, M. Lozupone ${ }^{2}$, F. Panza ${ }^{1}$, R. Sardone ${ }^{2}$, N. Quaranta ${ }^{3}$, G. Logroscino ${ }^{1,3}$

${ }^{1}$ Unit of Neurodegenerative Diseases, Department of Clinical Research in Neurology, University of Bari "A. Moro" at Pia Fondazione Card. G. Panico, Tricase, Lecce, Italy; ${ }^{2}$ IRCCS "Saverio De Bellis, Castellana Grotte, Bari, Italy; ${ }^{3}$ Department of Basic Medical Sciences, Neurosciences and Sense Organs, University of Bari "A. Moro", Bari, Italy

Objective: To study the association of peripheral and central presbyacusis with cardiovascular and dementia risk scores (RSs), in an elderly population.

Background: RSs are used to predict vascular and cognitive outcomes. There are no studies investigating the relationship between RSs and age-related hearing impairment.

Methods: Subjects enrolled in the GreatAGE study, a population based study on aging in subjects 65+ (Castellana Grotte, Southern Italy) underwent multidisciplinary assessment including neurological, neuropsychological, geriatric and otolaryngology examinations. Information about vascular and other risk factors were collected in a structured interview. Mild cognitive impairment (MCI) was diagnosed according to Petersen criteria; peripheral and central presbycusis according to Gates criteria. Two risk scores were considered: the Reitz RS, a late-life RS for the 4-year prediction of dementia; the Framingham general RS largely used for the 10-year prediction of cardiovascular events, but also proposed to predict dementia.

Results: In a sample of 947 individuals (530 males, 417 females; median age 73 years, range 65-92), 36.1\% were in the high-risk categories using the Reits RS and $27.8 \%$, using the Framingham RS. The Retz RS was associated with the presence of MCI ( $p<$ $0.0001)$ and central presbycusis $(\mathrm{p}<0.0001)$. The Framingham RS was associated only with the presence of central presbycusis ( $\mathrm{p}=$ 0.008), but not with MCI. None of the two RSs was associated with the presence of peripheral presbycusis.

Conclusions: The association between high vascular and dementia RS with cognitive impairment and central presbycusis may indicate that cognitive impairment and central presbycusis share the same pathogenic mechanism.

\section{6}

\section{Spatial Epidemiology of Amyotrophic Lateral Sclerosis in Piedmont and Aosta Valley, Italy: A Population-Based Cluster Analysis}

R. Vasta ${ }^{1}$, A. Calvo ${ }^{1,2}$, C. Moglia ${ }^{1,2}$, S. Cammarosano ${ }^{1}$, U. Manera $^{1}$, A. Canosa' , F. D'Ovidio' , L. Mazzini ${ }^{3}$, A. Chiò ${ }^{1,2}$

${ }^{1}$ ALS Center, 'Rita Levi Montalcini' Department of Neuroscience, University of Turin, Turin, Italy; ${ }^{2}$ Azienda Ospedaliero Universitaria Città della Salute e della Scienza, Turin, Italy; ${ }^{3}$ ALS Center, Department of Neurology, Azienda Ospedaliero Universitaria Maggiore della Carità, Novara, Italy

Objective: We aimed to perform a spatial analysis of a 20-years period Amyotrophic Lateral Sclerosis (ALS) cohort from the Piedmont and Aosta Valley ALS register (PARALS)

Background: The analysis of the spatial distribution of cases could give important cues on putative environmental causes of a disease. Indeed, analysis of the spatial distribution of cases could demonstrate clusters of higher or lower incidence. Subsequently, hypotheses about exposures potentially responsible for such clusters could be made.

Methods: Data from the PARALS were used. Address at the moment of diagnosis was considered for each ALS case. Municipalities' and census divisions' resident populations during the 1995-2014 period were obtained. Cluster analysis was performed adopting both Moran's Index and the Kulldorff's spatial scan statistic.

Results: A total of 2702 ALS patients were identified. Address was retrieved for 2671 (99\%) patients. Moran's Index was -0.01 ( $\mathrm{p}$-value $=0.83)$ thus revealing no clusters. SatScan identified no statistically significant clusters. When census divisions were considered, Moran's Index resulted 0.13 ( $\mathrm{p}$-value $=0.45$ ); SatScan revealed one statistically significant small cluster in the province of Alessandria. Here, 0.0099 cases were expected and 3 cases were observed (relative risk 304.60; 95\% CI 109.83-845.88, p-value 0.03).

Conclusion: Our study showed a substantial homogeneous distribution of ALS cases in Piedmont and Aosta Valley. The population-based setting and the adoption of proper statistical analyses strengthen the validity of our results. Such finding further suggests the involvement of multiple environmental and genetic factors in the ALS pathogenesis. 


\section{7 \\ Diagnostic Role of Biomarkers of Inflammation in Amyotrophic Lateral Sclerosis}

\begin{abstract}
Benmahamed Sara ${ }^{1,2}$, Rosanna Tortelli ${ }^{1}$, Chiara Zecca ${ }^{1}$, Marco Piccininni ${ }^{1}$, Vincenzo Brescia ${ }^{3}$, Giancarlo Logroscino ${ }^{1}$
\end{abstract}

${ }^{1}$ Unit of Neurodegenerative Diseases, Department of Clinical Research in Neurology, University of Bari Aldo Moro Ospedale Card. G. Panico, Bari, Italy; ${ }^{2}$ University of Limoges, France; ${ }^{3}$ Unit of Laboratory Medicine AO Card. G. Panico, Tricase, Italy

Objective: To evaluate plasma levels of inflammatory biomarkers in patients with amyotrophic lateral sclerosis (ALS) compared to control subjects, and to evaluate if a combination of different inflammatory biomarkers can be helpful in distinguishing between different clinical phenotypes of ALS.

Background: ALS is a progressive motor neuron disease leading to death of affected individuals within 2-3 years. The involvement of inflammation in the pathogenesis of ALS is increasingly recognized but still not well understood.

Methods: Plasma levels of five cytokines (IL2, IL6, IL10, IFNgamma and TNF-alpha) were measured in 79 patients with ALS, enrolled from a prospective population-based registry in Puglia (SLAP), Southern Italy, and in 79 age and sex matched healthy controls. Each patient underwent a neurological examination and a functional assessment. The cytokine analysis was performed using the Bioplex technology (Bio-Rad).

Results: All cytokines measured were found to be significantly increased in patients compared to controls $(\mathrm{p}<0.0001)$. The IL6 had the highest median concentration $(10.11 \mathrm{pg} / \mathrm{mL})$ in the ALS group. Furthermore using ROC analysis, it showed the highest diagnostic accuracy among the studied biomarkers. At a cut-off point of $5.71 \mathrm{pg} / \mathrm{mL}$, it was able to discriminate patients from controls with a good sensitivity and specificity $(91 \%$ and $87 \%$ respectively) and with a high AUC: 0.933. The IFN-gamma had the lowest median concentration $(1.13 \mathrm{pg} / \mathrm{mL})$ in cases. The only biomarker with a significant correlation with demographic and clinical variables was the IL6, that correlated positively with age $(r=$ $0.25 ; \mathrm{p}=0.025)$ and negatively with ALSFRSr at evaluation $(\mathrm{r}=$ $-0.32 ; \mathrm{p}=0.007)$ and MMT $(\mathrm{r}=-0.33 ; \mathrm{p}=0.004)$. There were no differences in biomarker levels between patients with bulbar or spinal onset of the diseases, neither between the different ALS phenotypes. Furthermore, no significant association was found between biomarker plasma concentrations and age, sex, ALSFRS-R at evaluation, site of onset and duration of disease from the symptom onset.

Conclusion: These results highlight the role of a panel of inflammatory markers as a diagnostic aid in ALS. In line with previous studies, this panel of plasma biomarkers could help in distinguish ALS cases from healthy controls. Further studies are needed to better clarify the role of inflammation in ALS.

\section{8}

\section{A Case-Control Study on Parkinson's Disease and Prior Exposure to General Anesthesia}

\author{
S. Mastrilli, D. Santangelo, A. Cinturino, V. Perini, F. Valentino, \\ P. Ragonese, G. Savettieri, M. D'Amelio
}

Università degli studi di Palermo - BioNec Department, Palermo, Italy

Objective: To evaluate the association between exposure to general anesthesia (GA) and Parkinson's disease (PD).

Background: Since 70s, animal and in-vitro studies suggested the potential neurotoxicity of general anesthetics. Few epidemiological studies explored the potential role of general anesthesia as risk factor for PD.

Methods: PD cases were matched for sex and age ( \pm 2 year) with at least one control. Demographic and clinical variables, together with data on general anesthesia exposure prior to PD onset, were gathered through detailed questionnaire and interview. Odds ratio, calculated by means of logistic regression analysis, was used as measure of association between exposure to general anesthesia and PD.

Results: A total of $251 \mathrm{PD}$ patients and 315 healthy controls were enrolled. A significant association was observed between PD and prior exposure to general anesthesia. Compared to controls, patients with PD were $50 \%$ more likely to have undergone general anesthesia before the onset of the disease (OR 1.5 [CI 95\% 1.042.03]; $\mathrm{p}=0.03)$. A positive significant trend $(\mathrm{p}=0.003)$ was observed with number of GA.

Conclusion: We observed an association between $\mathrm{PD}$ and prior exposure to general anesthesia. It is plausible to consider a neurotoxic effect of GA, hypothesis supported by the observed cumulative dose effect.

\section{9}

\section{Headache, Vascular Risk Factors and Dementia}

Tobias Kurth

Institute of Public Health, Charité Universitaetsmedizin Berlin, Berlin, Germany

Objective: To present recent data linking headache, in particular migraine, vascular risk factors, and dementia

Background: Migraine has been associated with several vascular risk factors and incident cardiovascular disease. As chronic conditions across many decades, it also has been hypothesized that headache or migraine lead to faster cognitive decline and dementia.

Methods: Data of population-based studies will be summarized and newest aspects presented.

Results: The association between migraine and vascular risk factors has been strengthened and migraine is now routinely considered in risk prediction models for cardiovascular disease. Nonmigraine headache does not carry this risk. Most studies do not support that individuals with migraine have a higher risk of dementia.
98

Neuroepidemiology 2018;50:74-103

DOI: $10.1159 / 000487692$ 15th Italian Congress of Neuroepidemiology, Tricase 2018 
Conclusions: Migraine should be considered a strong risk marker for increased prevalence of vascular risk and increased risk of cardiovascular disease. Dementia is not related to migraine.

\section{0}

\section{The Incidence of Dementia in the Very Old}

Ugo Lucca

Laboratory of Geriatric Neuropsychiatry, Department of Neuroscience, IRCCS Istituto di Ricerche Farmacologiche Mario Negri, Milan, Italy

Objective: To review epidemiological investigations in the oldest-old and discuss the methodological issues to face in performing studies on dementia in this age segment.

Background: Two-thirds of all dementia cases are 80 years and older and almost half 85 years or over. Due to increasing population aging, in a few decades persons with dementia in these age groups will be the vast majority of dementia cases. Estimates of dementia occurrence in the oldest-old are crucial for a better understanding of the heterogeneous pathological mechanisms underlying dementia and its relationship with age. However, conducting longitudinal studies in this population segment poses several challenges.

Methods: Literature databases were systematically searched with no date restrictions to identify studies on prevalence and incidence of dementia. We initially included in the review epidemiological investigations reporting estimates in the oldest-old and then focused on the subgroup of population-based studies.

Results: Most studies included very small numbers of oldestold often grouping them into age segments that are too broad $(80+, 85+, 90+)$. Estimates in the extreme ages of life were almost absent. Many studies were not population-based or were poorly representative of the general population. Several warnings about the difficulties of diagnosing dementia or conducting studies in the oldest-old are based on little evidence and seem overstated.

Conclusion: Exclusion of the oldest-old from research represents a bias that can affect conclusions based on findings in younger age groups. Individuals included in basic, clinical and epidemiological studies on dementia need to properly reflect the population at risk, "otherwise, research on dementia may be in danger of simply missing the point" (Shoenmaker and Van Gool 2004).

\section{1 \\ Cerebrovascular Diseases in a Sicilian Elderly Community: Results from a Population Based Study}

P. Ragonese, M. Riolo, G. Smorlesi, P. Aridon

Dipartimento di Biomedicina Sperimentale e Neuroscienze

Cliniche, Università di Palermo, Palermo, Italy

Objective: Our aim was to assess incidence and mortality rates of cerebrovascular disease, in a Sicilian population using data from a population-based survey of elderly participants.

Background: Stroke is one of the most disabling and burdensome health conditions worldwide.

Methods: A door-to-door survey was carried out in the city of Bagheria, Sicily (prevalence day September 30th, 2006). A cohort of 2,200 persons was randomly stratified, obtaining a $25 \%$ sample of the whole population aged 65 years or more. We obtained clinical data for the whole cohort after nine year from local Health Institution. Individuals were evaluated at baseline (2007-2008) and at the end of follow-up period (2016). We calculated crude, and age and sex specific incidence rates, as well as cause specific mortality rates, with $95 \%$ confidence intervals.

Results: We identified 176 incident patients with cerebrovascular disease during the follow up giving a total incidence of $888.9 / 100,000$ person years (CI: 888.86-888.94). Incidence rate was higher in men (1010.00, CI: 1009.94-1010.06) than women (790.30; CI: 790.24-790.36). Cause specific mortality rate for CVD was 353.54/100,000 (CI: 353.50-353.58) in the whol cohort, 420.12 in men (CI: 420.06-420.18) and 297.54 in women (CI: 297.48-297.60). Age-specific incidence rates of cerebrovascular disease increased with advancing age.

Conclusion: In the Bagheria Cohort study, incidence of cerebrovascular diseases increased with age. Stroke incidence rates and mortality were significantly higher for men compared to women. Our incidence rates provide new estimates for projection of future burden of disease in Italy and should be considered when planning prevention and stroke care services in this region.

52

\section{Cognitive Reserve and Its Role in Cognitive Decline}

Valentina Cardinali, Orietta Barulli ${ }^{2}$, Rosanna Tortelli ${ }^{2}$, Cristina Di Dio², Claudia dell'Aquila', Olimpia Difruscolo', Antonio Giannini ${ }^{3}$, Giuseppe Rinaldi', Giancarlo Logroscino ${ }^{2}$

${ }^{1}$ Department of Clinical Neurology and Neurophysiopathology, Hospital "Di Venere", Bari Italy; ${ }^{2}$ Unit of Neurodegenerative Disease, Department of Clinical Neurology, University of Bari "A. Moro", “Pia Fondazione C. Panico", Tricase, Italy; ${ }^{3}$ Department of Radiology, Hospital “Di Venere", Bari Italy

Objective: The objective of this investigation is to investigate the association between Cognitive Reserve (CR) and demographic, clinical and neuropsychological variables in a sample of patients with Subjective Memory Impairment (SMI), Mild Cognitive Impairment (MCI) and Alzheimer disease (AD). 
Background: CR is the capacity of an individual to cope brain damage caused by an advanced age or by an ongoing pathological process. CR is the results of education, profession, intellectual and social stimuli during lifetime.

Methods: cross-sectional multicentre study. Inclusion criteria: patients with diagnosis of mild AD, MCI and SMI. Neuropsychological assessment was used to assess the global cognitive and specific functions. The CR was assessed by Cognitive Reserve Index Questionnaire: four sections divided into the demographic data, education, work and leisure activities [Nucci, 2012].

Results: The age of patients ranged from 57 to 85 years and $90.6 \%$ was $>65$ aged. $42.35 \%$ received the diagnosis of $\mathrm{AD}, 47.06 \%$ MCI and $10.59 \%$ SMI. Compared to men, female patients showed to have lower scores in total CRI $(\mathrm{p}=0.001)$, CRI-Education $(\mathrm{p}=$ $0.02)$ and CRI-Work $(p<0.0001)$ but higher scores in CRILeisure. Patients with AD are women in $75 \%$ of cases. Usually, Women have less CR and then reduced compensation capacity to brain damage compared to men; for this reason females may be more vulnerable to neurodegeneration. Regarding the relationship between CR and age, in our results global, work and leisure CRI scores tend to decrease with age in the whole sample. The positive relationship between CRI-Education and age was demonstrated for women. This data could depend from a selection bias of the study: women with higher cultural knowledge could be more sensible to warn the incipient cognitive impairment and more available to participate in scientific researches. Investigating the relationship between CRI end neuropsychological variables the global CRI, along with CRI-Education and CRI-Work, is positively associated with global cognitive performances MMSE ( $\mathrm{r}$ : 0.300; 95\% CI: 0.09-0.48) and executive (Verbal Fluency test (r: $0.349,95 \%$ CI: 0.14 to 0.53 )) and visual-spatial functions (Rey Complex Figure Copy (r: $0.320 ; 95 \%$ CI: 0.11 to 00.50) the Trail Making Test A (r: $-0330,95 \%$ CI: -0.51 to -0.12$)$ ). Activities linked with high education and specificity responsibility jobs, such as repetition of specific skills or strengthen strategic cognitive skills, could stimulate brain networks and determine better performances to the neuropsychological tests.

Conclusion: The study demonstrates that the CR, extremely influenced by the age and gender, could influence cognitive performances. In this context, the knowledge of CR, acquired through the entire lifespan, can be useful to better understand the initial stages of the cognitive decline, which manifest themselves differently, depending on CR of each individual.

\section{3 \\ La Presa in Carico Delle Patologie Neurodegenerative Nel Territorio Puglese}

Vito Napoletano ${ }^{1,2}$

${ }^{1}$ ASL Bari, ${ }^{2}$ Neurology Territory National Group of the SIN (Italian Society of Neurology), Italy

The need to guarantee to the patient complex in discharge from the hospital/university departments a path that guarantees the taking charge; The planned PDTA will and will be able to foresee a territorial residency for the phase of sub-acuities and also residual assistance to the home care of complexity (the Territorial Assistance Centers - PTA). For the uninvolved person suffering from chronic neurodegenerative disease with disabling internal comorbidity (heart failure, COPD, Diabetes, IRC, etc.), the PDTA will and will be able to provide diurnal socio-health centers; the PAI (individualized care plan) will have to guarantee the complex, comorbid and fragile patient, the eventual scheduled activation of surgical day hospitals or dedicated day service at the hospital/ University facilities. The need to relate with the PTA (Territorial Assistance Centers) that represent the starting point for the integration between the different functions assistance and development of the Diagnostic Therapeutic Assistance Paths (PDTA), in order to achieve three main priority objectives:

A) to maximum supply efficiency (h24);

B) the best effectiveness, through the "pooling" of skills and abilities in an integrated form and shared by professionals through appropriate care pathways;

C) The greater appropriateness in the use of resources as they are coordinated and oriented towards shared health objectives;

D) Greater integration with the network of day-to-day and home-based health and social-health services.

People suffering from neurodegenerative diseases and families are often alone in the local realities. It is necessary to activate a diagnostic assistance path (PDTA) that integrates through the PTA with the Hospital and the University for the certain diagnostic aspects, possible and probable and that considers the follow-up with dedicated facilities (day service) with outpatient packages complex and coordinated (PACC) and also dedicated with diagnostic and treatment tools and for advanced medical-surgical devices. Also, the need to guarantee a global care in the area that considers the person in his environment: therapeutic-assistance aspects, aspects of reactivation and monitoring of functions, psycho-educational-social-welfare aspects, the latter very decisive in managing the factors contextual events often cause resistance to adherence to the PAI. Being able to follow patients suffering from neuromuscular pathologies and/or cardio-respiratory pathologies is one of the future challenges of Italian health care and being able to follow them from the outpatient stage to the home, is one of the strategic objectives of the National Health Plan and also means reducing the the cost of improper hospitalization, allowing, where possible, a better quality of life for patients and for as far as possible encouraged their stay at home.

Conclusion: Bi-directionality of interventions from the hospital to the territory and vice versa from the territory to the hospital for the realization of continuity of care.

- Level: company network MMG/PLS;

- II level: listening centers;

- III level: outpatient centers (university/hospital) and Day Service; or IV level: U.O. hospital for acute.

Activation of the Protected Resignation guidelines envisaged by the D.G. ASL Ba n. 1820/2014 (from Hospital to ADI, from Hospital to home rehabilitation, from Hospital to RSA or RSSA, from Hospital to SUAP, from Hospital to Hospice); U.O. hospital for post-acute intensive rehabilitation (identified by the code 56 , reserved for clinically unregulated patients, appropriate methods of access, Regulation by D.R.G. No 2067/2013); Extra-hospital nucleus for post-acute intensive residential rehabilitation (identified by Article 3 of Reg. Reg No. 12/2015, reserved for clinically stabilized adult or minor patients, access modalities foreseen by RR No. 12/2015); Extra-hospital nucleus for extensive residential
100

Neuroepidemiology 2018;50:74-103

DOI: $10.1159 / 000487692$ 15th Italian Congress of Neuroepidemiology, Tricase 2018 
rehabilitation (identified by Article 4 of the Delegated Reg. No. $12 / 2015$, reserved for clinically stabilized adult or minor patients, access modalities provided for by R. R. No. 12/2015); or V level: Extra-hospital nucleus for residential maintenance rehabilitation (identified by Article 5 of Reg. No. 12/2015, reserved for adult patients with high complexity of care, clinically stabilized, access modalities provided for by RR No. 12/2015); Extra-hospital core for semi-residential maintenance rehabilitation (identified by articles 6 and 7 of the Reg Regulating No. 12/2015, reserved for minor and adult patients access modalities foreseen by RR No. 12/2015 and its norms transitional); Socio-educational and rehabilitation day care center (identified by Article 60 of the Reg. No. 4/2007, which in turn was amended in the paragraph on the provision of Article 27 of Reg. No. 11/2015, which possible access to users "in conditions of severe disability").

54

\section{Time Trends in Population Studies of Dementia}

Walter A. Rocca

Department of Health Sciences Research and Department of Neurology, Mayo Clinic, Rochester, MN, USA

Objectives: In this invited lecture, I discuss time trends in the incidence of dementia.

Methods: It may be useful to consider the time trends for two groups of neurological diseases: 1) diseases that involve neurovascular mechanisms such as stroke, vascular, or mixed dementia, and 2) diseases that involve neurodegenerative mechanisms such as Alzheimer's disease, Parkinson's disease, and amyotrophic lateral sclerosis.

Results: My interpretation of the epidemiologic evidence is that the incidence of neurovascular diseases has declined, whereas the incidence of neurodegenerative diseases has increased in recent decades.

The incidence of stroke has declined in the United States and Canada; the incidence of parkinsonism has increased in a United States population and in Finland; and the incidence of amyotrophic lateral sclerosis has increased in Denmark and in Piemonte and Valle d'Aosta, Italy.

Declining time trends for the incidence of overall dementia have been reported from studies in the United States, the United Kingdom, and Canada. A recent study in Canada showed a significant decline in the incidence of dementia at older ages $(80+$ years; with a major neurovascular component), but a significant increase in incidence at younger ages (20-49 and 50-64 years; with a major neurodegenerative component). Therefore, the overall declining trend for dementia may conceal trends in opposite directions for the two major subtypes of dementia, the neurovascular and the neurodegenerative type.

Conclusions: The future of dementia remains somewhat unclear; however, we can be cautiously optimistic.

\section{5}

\section{Cognitive Training in Mild Cognitive Impairment:an Integrative Approach}

\author{
Lisa Dibello, Eriola Bagoj, Carlo Robusto, Mundi Ciro
}

Azienda Ospedaliera Universitaria O.O. R.R., Foggia, Italy

Objectives: To analyze cognitive rehabilitation efficacy in Mild cognitive Impairment patients.

Introduction: MCI is a transitional phase between the cognitive changes of a normal aging and mild dementia. This heterogeneous clinical condition leads patients to be considered "at risk" due to significant function losses that affect their life's quality.

Currently, no pharmacological treatments are available to improve symptoms or slow down the disease progression in patients with MCI. However, non-pharmacological intervention, like physical and cognitive training, can improve memory and executive functions in MCI. The best methods are memory training, psych-educational program and working memory treatment.

Methodology: Forty-six patients with a diagnosis of Mild Cognitive Impairment-amnesic (a-MCI) were recruited by the Neurology Department of the Ospedali Riuniti in Foggia to be subjected to a group cognitive training. The patients were randomly assigned into two groups: Experimental group (E.G., 23 patients) is subjected to a cognitive training and control group (Control group - C.G., 23 patients) not subjected to cognitive training. The E.G. performed a cognitive training for about six months; the C.G. did not perform any training but was recalled for neuropsychological follow-up.

Results: A multivariate analysis of repeated measures (RM MANOVA, Repeated-Measures Manova) between subjects was performed on a sample consisting of 46 subjects $(\mathrm{N}=46)$ with an average age of 73.48 randomly assigned to the experimental groups $(\mathrm{N} 0=23)$ and control groups $(\mathrm{N} 1=23)$. A significant difference of almost $17 \%$ was observed after training compared to control group, in which, on the contrary, was observed a decrease of $12 \%$ in neuropsychological follow-up.

The improvement was especially observed in episodic memory and in patient's mood as a result of cognitive training.

Conclusions: These results evidence that a targeted and holistic cognitive training involving also brain plasticity can lead to an improvement in cognitive functions in patients with Mild Cognitive Impairment. 
Abbrescia, D.I. 89, 95, 97

Addante, F. 80, 81

Alberti, D. 95

Alessandrini, F. 95

Alessio, G. 86

Alexa, V. 79

Andrea, D.A. 86

Annicchiarico, G. 87

Antonio, L. 86

Arcuti, S. 79, 96

Aridon, P. 99

Attolini, E. 87

Badin, F. 92

Bagoj, E. 101

Baldin, E. 78, 82, 92, 93

Barone, R. 77, 96

Barulli, M. 94

Barulli, M.R. 77, 86, 89, 96, 97

Barulli, O. 95, 99

Basedow-Rajwich, B. 75

Battaglia, M.A. 75

Battista, P. 77, 86, 94, 95, 96, 97

Bay, J. 75

Beghi, E. 82, 83, 85, 91

Beghi, M. 90, 91

Bellomo, A. 89

Benedetti, M.D. 76, 90

Bennett, D.A. 80

Beretta, S. 82

Bersano, E. 84

Bertuzzo, D. 84

Bianchi, A. 75

Bianchi, E. 82, 85, 91

Bisceglia, L. 87

Bisceglia, P. 80,81

Blanc, P.D. 84

Bogliun, G. 82

Bombardi, R. 95

Bonavina, G. 78

Bongianni, M. 91

Bonora, A. 95

Brayne, C. 77

Brescia, V. 77, 98

Calabrese, M. 76, 91

Calandra, G. 78

Calvo, A. 84, 97

Cammarosano, S. 84, 97

Canosa, A. 84, 97

Capozzo, R. 77, 86, 89, 94, 95, 96, 97
Capra, R. 95

Cardinali, V. 99

Carelli, V. 78

Carlà, R. 94

Carone, D. 82

Cascavilla, L. 80, 81

Casetta, I. 92, 93

Cereda, D. 82

Cevoli, S. 78

Chiesa, V. 91

Chiò, A. 83, 84, 97

Chiumento, G. 79

Cinturino, A. 98

Ciro, M. 101

Colombo, M. 82

Congedo, M. 90

Copetti, M. 79, 89, 96

Coppola, F. 86, 89, 95, 97

Cornaggia, C.M. 90, 91

Cortelli, P. 78

Cucovici, A. 79

Cugnasco, P. 84

D’Agostino, M. 81

Dagostino, M.P. 80, 81

D’Alessandro, R. 78

D’Alfonso, S. 79

Daltveit, A.-K. 82

D'Amelio, M. 98

Daniele, A. 85

De Donno, A. 94

De Filippis, G. 94

De Giorgi, A. 76

De Marchi, A. 93

De Pascali, S. 94

DeCarolis, P. 78

dell'Aquila, C. 99

Deotto, L. 76

d'Errico, A. 84

Di Dio, C. 86, 94, 95, 99

Di Lena, L. 97

Di Rosa, G. 91

Di Stasi, V. 78

Di Stolfo, G. 81

Dibello, V. 85

Dibello, L. 101

Difruscolo, O. 99

D’Onofrio, G. 80, 81

Donzelli, A. 90

D’Ovidio, F. 84, 97

Drulovic, J. 75

D’Urso, F. 89
Emanuele, R. 94

Erba, G. 91

Fabbri, G. 78

Facini, C. 85

Fanelli, R. 87

Farina, G. 76, 90

Fedele, A. 94

Ferrara, M. 79

Ferrara, R. 78

Ferrarese, C. 82

Ferrari, S. 95

Ferraro, D. 95

Fonti, C. 78

Forgione, A. 76

Forlivesi, S. 76, 90

Fornari, A. 75

Gabellini, A. 78

Gajofatto, A. 76, 91, 95

Gasparini, S. 82

Giannelli, G. 89

Giannini, A. 99

Giordano, A. 75

Giorgio, T. 86

Giulia, P. 86

Giussani, G. 82, 85, 91

Gobbin, F. 91, 95

Goldman, S.M. 84

Gorgoni, G. 87

Granieri, E. 92, 93

Grassano, M. 84

Grasso, A. 86, 89, 94, 95

Gravina, C. 80,81

Greco, A. 80, 81, 85

Griseta, C. 86, 94

Guarino, M. 78

Guerra, V. 95

Gullotta, B. 92

Ilardi, A. 84

Inchingolo, V. 81

Ivashynka, A. 79

Kleiter, I. 75

Kooij, L. 75

Koops, J. 75

Köpke, S. 75

Kurth, T. 98

La Morgia, C. 78

Laganà, A. 91
Lamanna, A. 89

Lancia, S. 76

Lauriola, M. 80, 81

Leo, A. 76, 87, 95

Leone, M. 81

Leone, M.A. 79

Liguori, R. 78

Lisnic, V. 79

Logroscino, G. $77,83,85,86$, $87,89,94,95,96,97,98,99$

Longo, M.G. 81

Lozupone, M. 85, 89, 94, 95, 97

Lozupone, T. 89

Lucca, U. 99

Magaudda, A. 91

Maggiore, G. 94

Mancinelli, C. 95

Manera, U. 84, 97

Mangiacotti, A. 80, 81

Marangi, A. 76, 90, 91, 95

Marcella, C. 86

Marchioretto, F. 76

Mariotto, S. 95

Mastrilli, S. 98

Mastroianno, S. 81

Mazzini, L. 84, 97

Mens, J. 75

Meza Murillo, E.R. 75

Mezzanzanica, M. 90

Miglietta, L. 87

Milanov, I. 75

Milo, R. 75

Mogavero, M.P. 77

Mogavero, P. 86

Moglia, C. 84, 97

Monaco, S. 91, 95

Mora, G. 84

Musarò, C. 77

Musio, M. 77

Myhr, K.-M. 92, 93

Naldi, P. 79

Napoletano, V. 100

Nassetti, S. 78

Oliver, D.J. 75

Orlandi, R. 95

Pacilli, M. 81

Padovano, G. 82

Pantieri, R. 78 
Panza, F. $80,81,85,86,89,94$, 95, 97

Paris, F. 80,81

Paroni, G. 80, 81

Patti, F. 75

Pekmezovic, T. 75

Perini, V. 98

Petrarolo, V. 87

Piccininni, M. 77, 86, 89, 94, 95, 96, 97, 98

Piccolo, B. 85

Pirovano, M. 82

Pisani, F. 85

Pisano, F. 84

Piscitelli, P. 94

Poletto, E. 91

Porro, G. 91

Provinciali, L. 88

Pugliatti, M. 82, 92, 93

Pupillo, E. 83

Quaranta, N. 86, 89, 95, 97

Racovita, A. 79

Ragonese, P. 75, 98, 99
Rahn, A.C. 75

Raho, A.M. 94

Realmuto, S. 75

Reindl, M. 95

Ricceri, F. 84

Riise, T. 82, 92, 93

Rinaldi, G. 99

Rinaldi, R. 78

Riolo, M. 99

Robusto, C. 101

Rocca, W.A. 101

Ronzoni, G. 90

Rossi, F. 95

Russo, A. 81

Russo, M. 91

Sacquegna, T. 78

Salemi, G. 75

Sambati, R. 96

Samoggia, G. 78

Sancarlo, D. 80, 81

Santangelo, D. 98

Sara, B. 98

Sardone, R. 85, 86, 89, 94, 95,97
Sastre-Garriga, J. 75

Savettieri, G. 75, 98

Sborgia, G. 86

Scaglione, C. 78

Scanziani, S. 82

Scianatico, G. 77

Seripa, D. 80, 81, 85, 89, 95

Silber, E. 75

Simone, I.L. 96

Sirbu, N. 79

Smorlesi, G. 99

Solari, A. 75

Stallone, R. 85, 94

Stella, E. 89

Stenta, G. 76

Stivanello, E. 78

The PRO-LONG Study Group 82

Tondo, D. 94

Tortelli, R. 77, 86, 89, 94, 95, 96, 97, 98, 99

Trentini, C. 82

Turatti, M. 91, 95

Tursi, M. 86
Urbano, M. 80, 81

Valentino, F. 98

Valentino, M.L. 78

Vasta, R. 97

Vazzoler, G. 75

Vecchio, D. 79

Veronese, S. 75

Vianello, M. 76

Vicenzi, V. 76, 90

Vignatelli, L. 78, 88

Vittadini, G. 90

Voltz, R. 75

Vosburgh, J. 75

Wolfson, C. 92, 93

Zanchi, C. 82

Zanoni, M. 91

Zarrelli, M. 79

Zecca, C. 77, 96, 98

Zenesini, C. 78

Zoccarato, M. 95

Zuliani, C. 95

Zuliani, L. 95 